\title{
Empaquetamiento e incentivos para mejoras en calidad
}

\author{
Correa, Alexander \\ Fundación Universitaria Los Libertadores (Colombia) \\ Correo electrónico: alexander.correa@libertadores.edu.co
}

\begin{abstract}
RESUMEN
Este estudio presenta la competencia más allá de un solo mercado producto donde una firma multiproducto compite con rivales monoproducto mediante el uso de diferentes estrategias de empaquetamiento que impactan los incentivos de las firmas por realizar mejoras de calidad en diferentes modos. La estrategia de empaquetamiento puro puede hacer que la firma multiproducto invierta en mejoras de calidad cuando los costos asociados son comparativamente bajos y la utilidad adicional de la mejora de calidad es relativamente alta, pero desalienta a las firmas monoproducto a mejorar la calidad. En el caso de empaquetamiento mixto, este resultado inevitablemente sucede en el mercado más competitivo y probablemente se encuentra en el mercado menos competitivo cuando los mercados no son tan diferentes en intensidad competitiva. Por lo tanto, ambas estrategias de empaquetamiento afectan el excedente del consumidor cuando los dos mercados son significativamente diferentes en la intensidad de la competencia debido a la influencia negativa de las distorsiones del mercado después de interrelacionar los dos mercados.
\end{abstract}

Palabras clave: empaquetamiento, mejoras en calidad, telecomunicaciones, regulación. Clasificación JEL: L13; L41; L51.

MSC2010: 00-02; 39-02. 


\title{
Packaging and quality improvements
}

\begin{abstract}
This study presents the competition beyond a single product market where a multi-product firm competes with monoproduct rivals through the use of different bundling strategies that impact the firms' incentives to make quality improvements in different ways. The pure bundling strategy can make the multiproduct firm invest in quality improvements when the associated costs are comparatively low, and the additional utility of quality improvement is relatively high, but it discourages single-product firms from improving quality. In the case of mixed bundling, this result inevitably happens in the most competitive market, and is probably found in the less competitive market when markets are not so different in competitive intensity. Therefore, both bundling strategies affect the consumer surplus when the two markets are significantly different in the intensity of competition due to the negative influence of the market distortions after interrelating the two markets.
\end{abstract}

Keywords: packaging, quality improvements, telecommunications, regulation.

JEL classification: L13; L41; L51.

MSC2010: 00-02; 39-02.

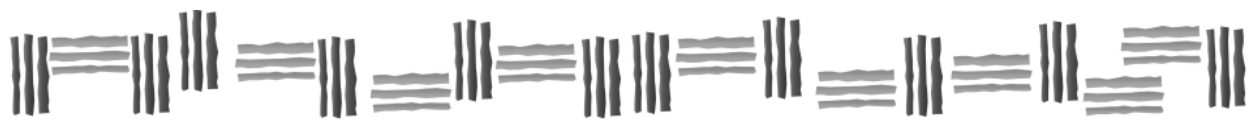




\section{Introducción.}

La convergencia digital ha creado incentivos para que los operadores de telecomunicaciones expandan sus modelos de negocio y ofrezcan diferentes servicios todos bajo una misma marca. Por ejemplo, en Colombia, diferentes operadores de telecomunicaciones ofrecen paquetes triple play que incluyen TV por cable, banda ancha y telefonía fija. El operador aprovecha la lealtad de marca y ofrece descuentos atractivos a los consumidores por comprar el producto empaquetado. Los operadores multiproducto tienen una mayor probabilidad de ser dominantes ya que cuentan con más estrategias para competir que sus rivales monoproducto. Por ejemplo, cuando la competencia se da en precios, un operador multiproducto puede usar la estrategia de empaquetamiento para aumentar sus beneficios y aumentar su participación de mercado, en tanto que un operador monoproducto probablemente vea reducidos sus beneficios y consumidores. Si la estrategia de empaquetamiento es extremadamente agresiva, los operadores pequeños tienen una alta probabilidad de salir del mercado. Desde el punto de vista de la demanda, aunque algunos consumidores que compran el producto empaquetado se benefician de los descuentos, el hecho de que los precios individuales presenten distorsiones puede a afectar a otros consumidores, especialmente a los usuarios monoproducto (Armstrong, 2011; Reisinger, 2006) reduciendo el excedente del consumidor agregado (Gans \& King, 2006; Granier \& Podesta, 2010; Reisinger, 2006; Rennhoff \& Serfes, 2009). En el contexto de telecomunicaciones con el rápido crecimiento de tecnologías de última generación, el principal enfoque que se le da al tema del empaquetamiento no solo es de los precios individuales distorsionados, sino también el de los incentivos que se generan para implementar mejoras de calidad sobre los productos ofrecidos. Sin embargo, gran parte de la literatura existente solo enfatiza los efectos del empaquetamiento en un contexto de competencia en precios. Solo pocos estudios extienden las decisiones de las firmas a otros aspectos en el contexto de empaquetamiento, por ejemplo, elecciones de calidad y reducción de costos en I+D (Avenali, D’Annunzio \& Reverberi, 2013; Choi, 2004; Krämer, 2009).

Desde un punto de vista más amplio, el presente estudio contribuye a la literatura existente a través de un modelo en el que los operadores en el mercado no solo compiten en precios, sino también realizan distintas decisiones de inversión sobre las características de calidad del producto (por ejemplo, una compañía de internet de banda ancha puede mejorar su estabilidad de conexión o el servicio al cliente para hacer su servicio comparativamente más atractivo que el servicio de sus competidores.).

Los operadores actuando en dos mercados duopólicos pueden inicialmente elegir sus propios niveles de mejora de calidad para posteriormente fijar precios (en este contexto, el tema de calidad no se relaciona con diferenciación vertical en calidad, donde los consumidores tienen diferentes preferencias por calidad de producto. En realidad, este modelo está basado en diferenciación horizontal donde los consumidores tienen diferentes gustos por un producto específico ofrecido por una firma en particular). La situación se modifica porque un operador monoproducto tiene ahora herramientas adicionales para responder a las estrategias agresivas del empaquetamiento del operador multiproducto. En un escenario de empaquetamiento puro, comparado con la situación donde no existe empaquetamiento, los incentivos del operador monoproducto para implementar mejoras de calidad se reducen. Entre tanto, en parte debido a su preocupación por la externalidad existente entre mercados, el operador multiproducto aumenta sus niveles de mejora de la calidad si dicho proceso es lo suficientemente costo eficiente en términos de generación de utilidad adicional. En el mercado monoproducto, el operador menos competitivo se ve obligado a reducir su precio, mientras que en el mercado más competitivo puede aumentar su precio cuando se beneficia significativamente de una reducción sustancial en la intensidad de la competencia con un número limitado de elecciones de producto que se da como consecuencia del empaquetamiento puro. Bajo empaquetamiento mixto, el operador multiproducto ofrece un descuento en el paquete en línea con los resultados estándar sobre fijación individual de precios (Armstrong, 2011; Reisinger, 2006). En el mercado más competitivo, se promueven las mejoras en la calidad de la firma multiproducto en contraste con el deterioro presentado por su contraparte monoproducto. Un resultado parecido se encuentra en mercados menos competitivos cuando los dos mercados son similares en términos de intensidad competitiva. Es posible que ambas estrategias de empaquetamiento reduzcan el excedente del consumidor cuando los dos mercados son significativamente diferentes en intensidad competitiva. En este caso, la distorsión ineficiente sobre el 
consumidor predomina y el regulador debería monitorear la implementación de estrategias de empaquetamiento, especialmente cuando un mercado más competitivo está apalancado con uno menos competitivo. Sin embargo, las estrategias de empaquetamiento pueden ser un incentivo para implementar mejoras de calidad en algunas situaciones.

\section{Revisión de la literatura.}

La literatura existente analiza los resultados de mercado de las estrategias de empaquetamiento en diferentes situaciones de acuerdo al grado de competencia y de correlación en valor de los productos. En un marco de duopolio simétrico, la correlación de las valoraciones desempeña un papel importante. Bajo una correlación negativa, los consumidores son más homogéneos cuando ellos consideran comprar productos empaquetados en vez de productos individuales. El efecto "robo de negocios" domina el efecto ordenador del empaquetamiento. El efecto robo de negocios, business stealing en inglés, se refiere al efecto (negativo) sobre la demanda de los competidores cuando una firma realiza algún tipo de acción (generalmente en relación a la fijación de precios, pero podría ser cualquier elección de una variable estratégica de la empresa). Esto es opuesto a aumentar la demanda a través de consumidores que ya compran de la empresa, o atrayendo nuevos consumidores al mercado. Los beneficios de los operadores se reducen debido al dilema del prisionero que se da cuando ambos operadores eligen vender productos empaquetados y no productos individuales.

De otro lado, el resultado opuesto aplica cuando la situación es de correlación positiva de valoraciones (Reisinger, 2006). Esto puede aplicar a la situación de una fusión entre operadores monoproducto homogéneos bajo la correlación positiva de valoraciones en un modelo circular (Granier \& Podesta, 2010) y eventos en los que las firmas competidoras "coluden" para ofrecer productos empaquetados que les permitan extraer la renta de los consumidores, situación que no se daría si decidieran competir en precios (Armstrong, 2011). Sin embargo, una vez dado el empaquetamiento, los precios individuales aumentan a pesar de que el producto empaquetado experimenta un descuento significativo (Armstrong, 2011; Reisinger, 2006). Adicionalmente, los operadores "persuaden" a los consumidores a través de sus estrategias a que éstos compren el producto empaquetado y no la combinación de productos individuales que eventualmente puede ser preferida por los consumidores. Debido a que puede ser usada como herramienta que los beneficios de los operadores pero que provoca ineficiencias distributivas, el empaquetamiento como estrategia puede ir en contra del bienestar del consumidor (Gans \& King, 2006; Granier \& Podesta, 2010; Reisinger, 2006; Rennhoff \& Serfes, 2009) e incluso del bienestar social (Granier \& Podesta, 2010).

Adicionalmente, en un contexto de competencia duopólica asimétrica, y debido a las estrategias de empaquetamiento, un operador multiproducto está en una mejor posición que un operador monoproducto. Nalebuff (2004) señala que la estrategia de empaquetamiento es una estrategia creíble para la empresa establecida en el mercado multiproducto para aumentar sus beneficios sin importar la decisión de entrada de una firma nueva. Este resultado está en línea con Choi (1996, 2004), donde se apoya la teoría de apalancamiento donde la empresa establecida en el mercado transfiere su poder de mercado del mercado monopolístico al mercado duopolistico. La estrategia de empaquetamiento es más razonable que la estrategia de limitar precios que obliga a la empresa establecida en el mercado a reducir sus beneficios. Gans y King (2006) encuentran que, en su modelo extendido de un operador integrado y dos operadores independientes, el bienestar social se reduce debido a la ineficiencia que se da en los productos asignados al consumidor a pesar de que no haya una estrategia de empaquetamiento por parte de los operadores independientes monoproducto. En contraste, si se permite que dos operadores independientes se fusionen, ellos lo harán, pero finalmente los dos operadores integrados no emplearán estrategias de empaquetamiento con el fin de minimizar la competencia. Como consecuencia se observará incrementos en bienestar social que lo que se daría en el caso de operadores independientes aliados compitiendo con productos empaquetados. 
En la introducción de un grupo de consumidores monoproducto con el supuesto de ahorro en costos para los consumidores multiproducto que obtienen por realizar de "compras de una sola parada", Thanassoulis (2007) concluye que bajo preferencias específicas a la firma, las firmas pueden aumentar su beneficio mediante el cobro de mayores precios individuales a este grupo de consumidores monoproducto comparado con la situación de no empaquetamiento y al mismo tiempo ofrecer un descuento en el producto empaquetado para atraer los consumidores multiproducto cuya demanda es más elástica que la de los consumidores monoproducto. Bajo preferencias específicas al producto (paquetes híbridos están disponibles), un dilema del prisionero ocurre cuando las firmas deciden vender productos empaquetados a pesar de que se presenten caídas en los beneficios. Como resultado, el excedente del consumidor bajo preferencias específicas a la firma se reduce, pero el excedente del consumidor bajo preferencias específicas al producto aumenta producto del empaquetamiento.

En el modelo extendido de diferentes grados de diferenciación de producto en dos mercados, Thanassoulis (2011) encuentra que, bajo convergencia parcial, la firma fusionada puede aumentar sus beneficios, aunque no ofrezca un descuento sobre el producto empaquetado. Los precios individuales del mercado más competitivo aumentan, pero los de los mercados menos competitivos se reducen debido a la estrategia de empaquetamiento que impacta de manera adversa a los consumidores monoproducto. A los consumidores multiproducto se les cobra un precio mayor, pero el excedente del consumidor de este grupo de consumidores es ambiguo de acuerdo al grado de competencia de los mercados. Bajo convergencia total, las dos firmas fusionadas bilateralmente deciden vender paquetes por lo que los productos empaquetados son ahora menos diferenciados debido a una reducción de los costos de degustación. Como resultado de una mayor competencia, ambas firmas fusionadas deciden ofrecer descuentos sobre el paquete. Comparado al escenario donde no existe convergencia, los precios individuales permanecen inalterados, pero los consumidores multiproducto disfrutan de descuentos competitivos sobre el producto empaquetado. En general, el excedente agregado del consumidor aumenta bajo un escenario de convergencia total. La firma fusionada en el escenario de convergencia total tiene menores beneficios que las firmas unilaterales fusionadas en el escenario de convergencia parcial. En contraste al resultado de Granier y Podesta (2010) y Reisinger (2006) en juegos de dos etapas donde las firmas pueden decidir sobre si ofrecen un descuento por paquetes, la primera fusión está en una ventaja distinta pero sus rivales deciden permanecer independientes para poder minimizar la competencia.

El empaquetamiento también influeye en los incentivos de la firma para invertir en investigación y desarrollo. Las firmas multiproducto con estrategias de empaquetamiento invierten más en $\mathrm{I}+\mathrm{D}$ en reducción de costos e innovación mientras que sus rivales monoproducto estratégicamente reducen sus inversiones (Choi, 2004; Heeb, 2003). En un escenario más amplio de diferenciación vertical, las firmas ofrecen diferentes niveles de calidad para llegar a diferentes grupos de consumidores (Tirole, 1988; Wauthy, 1996). Bajo un escenario de empaquetamiento, una firma multiproducto puede transferir su poder de mercado de un mercado a otro mercado duopolista mediante la estrategia de empaquetamiento puro. Esta firma se convierte en dominante y vende a los consumidores de alta calidad. Sus beneficios son más altos que de los rivales monoproducto que tratan de evitar la competencia atendiendo a los consumidores de baja calidad (Avenali, D’Annunzio \& Reverberi, 2013; Krämer, 2009). Desde una perspectiva más amplia en inversión en calidad, el empaquetamiento puede tener efectos benéficos sobre el bienestar social en ciertas circunstancias (Avenali, D’Annunzio \& Reverberi, 2013). En el mundo de negocios, las firmas atraen a consumidores mediante ofertas de diferentes tipos cuando la competencia se intensifica. Es necesario analizar la pregunta de los efectos que tienen diferentes estrategias de empaquetamiento sobre el bienestar en escenarios de fijación de precios para poder determinar si el regulador es capaz de hacer una valoración exhaustiva y cuidadosa de las estrategias de empaquetamiento y sus efectos sobre el bienestar.

Este estudio está organizado como sigue. El modelo de dos mercados duopolistas es detallado en la sección 2. El análisis de los resultados de mercado después de empaquetamiento se encuentra en la sección 3. Finalmente, la sección 4 contiene las conclusiones y algunas recomendaciones para los reguladores. 


\section{Modelo.}

Existen una firma multiproducto y dos firmas monoproducto en dos mercados duopólicos. En el mercado A, la firma multiproducto compite con una firma monoproducto. En el mercado B, compite con la otra firma monoproducto.

Figura 1. Los dos mercados duopolisticos.

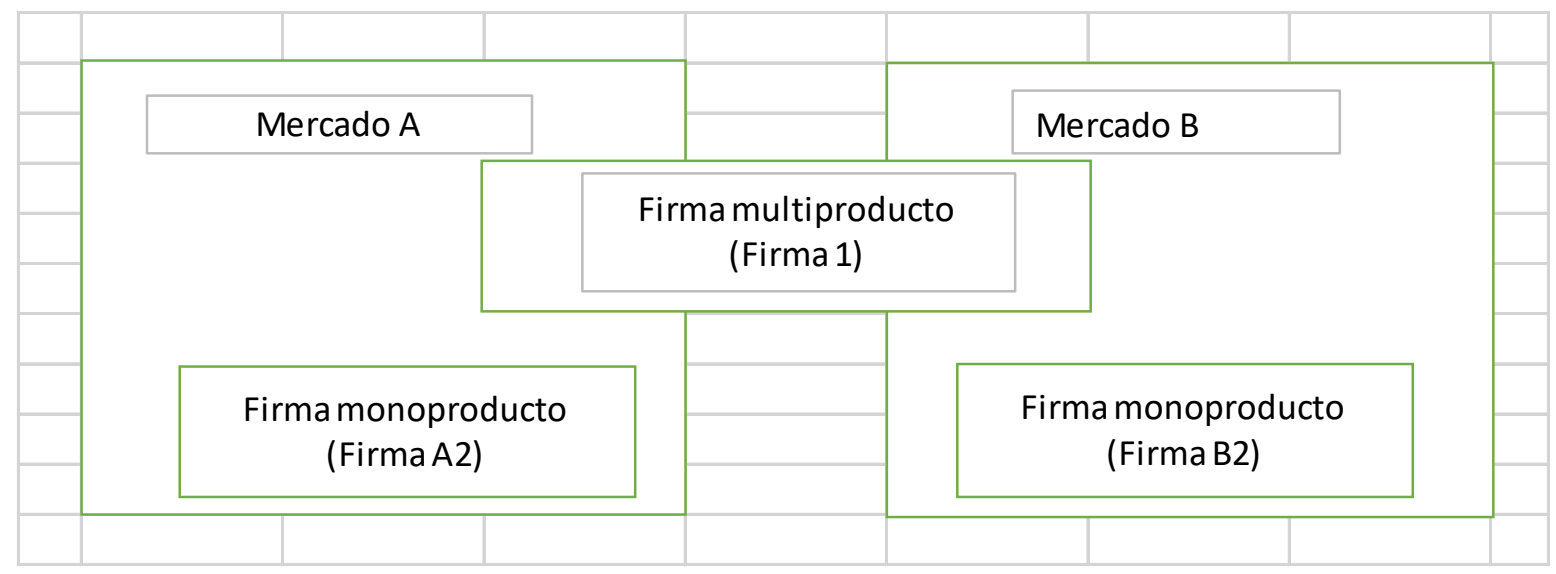

Fuente: Elaboración propia.

En cada mercado existen dos productos diferenciados horizontalmente por cada par de firmas competidoras (la diferenciación se da en productos no en firmas). La firma 1 tiene presencia en ambos mercados. Como se aprecia en la Figura 1, la firma 1 ofrece el producto A1 y el producto B1 para competir con las firmas A2 y B2 en los mercados A y B respectivamente. Los productos A y B pueden ser consumidos de manera independientemente (este modelo se enfoca en servicios de telecomunicaciones tales como TV por cable e internet de banda ancha. A diferencia de bienes informáticos, por ejemplo, computadores y software, estos servicios pueden ser consumidos separadamente.). Bajo un supuesto de demanda unitaria, cada consumidor puede comprar solo una unidad del producto A y una unidad del producto B (este supuesto puede ser aplicado a muchos servicios de telecomunicaciones. Por ejemplo, una familia normalmente se suscribe de manera mensual a un único proveedor de servicios para que le proporcione un paquete de telecomunicaciones tal como internet, cable, telefonía fija o móvil.). La firma 1 puede implementar estrategias de empaquetamiento para atraer consumidores en ambos mercados.

\section{Firmas}

La entrada al mercado ya ha ocurrido por parte de las firmas y los productos ofrecidos satisfacen los requerimientos mínimos de calidad impuestos por el regulador (los reguladores de telecomunicaciones monitorean permanentemente la calidad de los servicios de telecomunicaciones). De esta manera, los costos fijos de productos estándar son hundidos y se asume que son cero. Por simplicidad, se asume que el costo marginal constante del producto estándar es cero e igual para todas las firmas. Los servicios de telecomunicaciones incurren en costos fijos significativos en infraestructura, en tanto que los costos marginales son relativamente bajos. Adicionalmente, el costo marginal generalmente se calcula en términos constantes. Básicamente, las firmas compiten en precios. $P_{k i}$ es el precio unitario del producto $k i ; k \in\{A, B\}$ e $i \in\{1,2\}$.

Adicional a la competencia en precios, el modelo permite que las firmas diferencien sus productos a través de mejoras en la calidad de los mismos. Este modelo endogeniza las mejoras de calidad (éste no es un tema de diferenciación vertical en calidad). Es decir, cada consumidor percibe las 
mejoras de calidad y recibe una utilidad adicional como resultado de la calidad mejorada del producto. $\beta_{k i}$ es un nivel de mejora de calidad que una firma agrega a su producto estándar $k i ; k \in\{A, B\}$ e $i \in$ $\{1,2\}$. Este modelo está enfocado solo en $\beta_{k i}>0$. Reducir la calidad desde el mínimo estándar no es práctico, especialmente en mercados que son monitoreados de manera cercana por los reguladores. Las firmas incurren en costos adicionales de inversión asociados con el nivel de mejora de calidad que ellas elijan. $I_{k}\left(\beta_{k i}\right)$ es la función de costo de inversión en mejoras de calidad al nivel $\beta_{k i}$ en el mercado $k$. La función de costos de inversión es convexa en el nivel de mejoras de calidad.

$$
I_{k}\left(\beta_{k i}\right)=b_{k} \beta_{k i}^{2}
$$

$b_{k}$ es constante y positivo. Adicional a los costos de inversión, las mejoras en calidad de una firma pueden llevar a cambios en su costo marginal de producción. Se asume que las firmas tienen costos marginales de producción constantes $(\mathrm{cm})$. Debido a costos marginales de producción de cero en el producto estándar, el costo marginal positivo es una consecuencia directa de la mejora en calidad, i.e. $\mathrm{cm}=0+C_{k}\left(\beta_{k i}\right)=C_{k}\left(\beta_{k i}\right)$. Se asume que $C_{k}\left(\beta_{k i}\right)$ es una función lineal del costo marginal adicional de la mejora en el nivel de calidad $\beta_{k i}$ del producto $k i$ :

$$
C_{k}\left(\beta_{k i}\right)=c_{k} \beta_{k i}
$$

$c_{k}$ es constante y positiva. Por ejemplo, una empresa de banda ancha puede agregar un servicio de wifi gratuito en lugares públicos para sus consumidores que ya tienen el servicio de banda ancha en casa. La compañía de banda ancha tiene que invertir en costos adicionales de inversión para proveer el servicio público de wifi e incurrir en costos marginales más altos. Asimismo, si una firma elige una mejora alta para su producto, cada unidad vendida tiene costos marginales de producción altos debido a los costos marginales positivos implicados por la mejora de calidad. Por simplicidad, la función de costo de inversión y la relación entre mejoras en la calidad y los costos marginales de producción son idénticos entre firmas en el mismo mercado. Las firmas pueden tener diferentes funciones de costos de mejoras de calidad porque tienen diferentes tecnologías, experiencias y conocimiento tácito (know how). Adicionalmente, para atraer consumidores, las firmas posiblemente usan diferentes métodos para aumentar el valor de sus productos. Sin embargo, en una primera etapa de este estudio, se asume que el proceso de mejora de calidad es de conocimiento común por lo que la firmas tienen las mismas funciones de costos de mejoras de calidad.

\section{Consumidores}

Cada consumidor compra tanto una unidad de producto A como una unidad de producto B (demanda unitaria). Algunos estudios endogenizan los usuarios livianos que compran tanto el producto A como el producto B. En este estudio, solo los usuarios pesados que compran ambos productos están enfocados porque ellos desempeñan un papel crucial en la fijación del empaquetamiento de productos.

Basándose en el modelo de Hotelling, los consumidores están uniformemente distribuidos en un cuadrado unitario $[0,1]$. La combinación pura de [A1B1] y $[A 2, B 2]$ está localizada en $(0,0)$ y $(1,1)$ respectivamente. En tanto que la combinación hibrida de $[\mathrm{A} 1, \mathrm{~B} 2]$ y $[\mathrm{A} 2, \mathrm{~B} 1]$ está localizada en $(0,1)$ y $(1,0)$ respectivamente. El número de consumidores se normaliza a 1.

Un consumidor tiene una preferencia dada por un producto en particular dependiendo de la distancia entre su ubicación y la ubicación del producto. Un consumidor incurre en una desutilidad cuando el producto que ha escogido no es exactamente su producto ideal, que debería estar en la misma ubicación donde está el consumidor. $t_{k}$ es el costo de degustación que se refiere a la desutilidad por distancia en el mercado $k$. El costo de degustación es equivalente al costo de transporte en el modelo de Hotelling, que inversamente representa el grado de sustituibilidad entre dos productos rivales en el mercado. Se asume que el mercado A es más competitivo que el mercado B. $t_{A}=\theta t ; t_{B}=t ; \theta \in$ $(0,1)$. Cuando el costo de degustación se reduce, los dos productos diferenciados en el mercado son más sustituibles. Así que, el mercado es más competitivo en el aspecto de sustituibilidad. $V_{k}$ es la utilidad bruta del producto estándar k, que es idéntica entre consumidores. $V_{k}$ es lo suficientemente alta 
para garantizar que cada consumidor participa en el mercado y la demanda es atendida por las firmas. Cuando una firma elige la mejora de calidad del producto $k_{i}$ al nivel $\beta_{k i}$, los consumidores perciben dicha mejora de calidad y reciben una utilidad adicional $v_{k}\left(\beta_{k i}\right)$ del producto $k i$.

$$
v_{k}\left(\beta_{k i}\right)=\alpha_{k} \beta_{k i}
$$

$\alpha_{k}$ es una constante positiva. Se asume que $\alpha_{k}>c_{k}$. Con este supuesto, un nivel positivo de mejora de calidad existe.

Como ya se explicó, los consumidores compran tanto el producto A como el producto B. En el modelo inicial, se asume que consumir una combinación de producto $\mathrm{A}$ y del producto $\mathrm{B}$ resulta en una utilidad bruta aditiva, V_A+V_B. Sin embargo, debido al soporte técnico, diferentes tipos de servicios de telecomunicaciones son parcialmente (de manera imperfecta) sustituibles desde el punto de vista de los consumidores. De esta manera, la utilidad bruta de consumir una combinación de producto puede ser subaditiva (Armstrong, 2011; Venkatesh \& Kamakura, 2003). Por ejemplo, aunque los servicios móviles son obviamente distintos de comunicaciones vía internet, la tecnología permite que los usuarios de internet se comuniquen a través de software tal como Skype o chat como WhatsApp. En ese sentido, los servicios móviles y el internet de banda ancha pueden ser parcialmente sustituibles especialmente en un ambiente de convergencia digital. No obstante, por simplicidad, este estudio asume utilidad bruta aditiva de la combinación de los productos.

En este modelo, aunque un consumidor decida comprar ambos productos de la firma 1, no existen economías de alcance en el consumo conjunto de los productos. Por lo tanto, los beneficios en ahorros por pagar una sola factura o realizar compras de "una sola parada" triviales y omitidos por simplicidad.

Un consumidor, que está ubicado en $(x, y)$ en un cuadrado unitario $[0,1]^{2}$, enfrenta las siguientes cuatro elecciones como se ilustra en la Figura 2.

Figura 2. Consumidores en el modelo de dos mercados con diferenciación de producto horizontal.

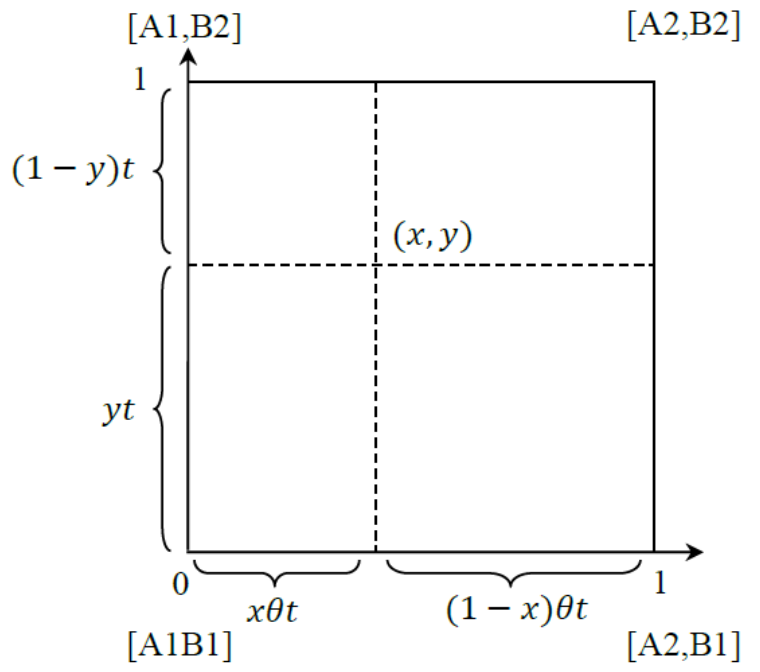

Fuente: Elaboración propia.

(I) $\quad[\mathrm{A} 1 \mathrm{~B} 1]$ de firma 1

Su utilidad neta de consumir producto A1 y B1 es

$$
V_{A}+V_{B}+\alpha_{A} \beta_{A 1}+\alpha_{B} \beta_{B 1}-x \theta_{t}-y t-P_{A 1 B 1}(\mathrm{I})
$$


(II) $[\mathrm{A} 1 \mathrm{~B} 2]$ de firmas separadas

Su utilidad neta de consumir producto A1 y B2 es

$$
V_{A}+V_{B}+\alpha_{A} \beta_{A 1}+\alpha_{B} \beta_{B 2}-x \theta_{t}-(1-y) t-P_{A 1}-P_{B 2}(\mathrm{II})
$$

(III) $[\mathrm{A} 2 \mathrm{~B} 1]$ de firmas separadas

Su utilidad neta de consumir producto A2 y B1 es

$$
V_{A}+V_{B}+\alpha_{A} \beta_{A 2}+\alpha_{B} \beta_{B 1}-(1-x) \theta_{t}-y t-P_{A 2}-P_{B 1}(\mathrm{III})
$$

(IV) [A2B2] de firmas separadas

Su utilidad neta de consumir productos A2 y B2 es

$$
V_{A}+V_{B}+\alpha_{A} \beta_{A 2}+\alpha_{B} \beta_{B 2}-(1-x) \theta_{t}-(1-y) t-P_{A 2}-P_{B 2}(\mathrm{IV})
$$

Se asume que la utilidad bruta de una combinación de producto es aditiva $\left(V_{A}+V_{B}\right) . P_{A 1 B 1}$ es el precio total de la combinación [A1B1]. La firma 1 ofrece $P_{A 1 B 1}$ de acuerdo a su estrategia de empaquetamiento.

- $\quad$ En el caso sin empaquetamiento, $P_{A 1 B 1}=P_{A 1}+P_{B 1}$

- $\quad$ En el caso de empaquetamiento puro, $P_{A 1 B 1}=\tilde{P}$ (el precio de un paquete puro [A1B1])

- $\quad$ En el caso de empaquetamiento mixto, $P_{A 1 B 1}=P_{A 1}+P_{B 1}-\delta$, donde $\delta$ es el descuento por adquirir el producto empaquetado.

Como puede ser visto en la Figura 2, el consumidor ubicado en $(x, y)$ compara su utilidad neta de las elecciones disponibles de (I)-(IV) y finalmente elige la combinación de producto que resulta en la utilidad neta más alta. En este modelo, la firma multiproducto tiene tres diferentes estrategias de empaquetamiento.

\section{(1) Sin empaquetamiento.}

La firma 1 ofrece los productos separadamente a los precios $P_{A 1}$ y $P_{B 1}$. Todas las cuatro elecciones (I) - (IV) están disponibles. Así, las participaciones de mercado están determinadas por el consumidor marginal en cada uno de los mercados. $\hat{x}$ y $\hat{y}$ denotan las ubicaciones de los consumidores marginales en los mercados A y B respectivamente. Los consumidores marginales en los mercados A y B resuelven las siguientes condiciones, correspondientemente:

$$
\begin{gathered}
V_{A}+\alpha_{A} \beta_{A 1}-\hat{x} \theta t-P_{A 1}=V_{A}+\alpha_{A} \beta_{A 2}-(1-\hat{x}) \theta t-P_{A 2} \\
\hat{x}=\frac{1}{2}+\frac{1}{2 \theta t}\left(P_{A 2}-P_{A 1}+\alpha_{A} B_{A 1}-\alpha_{A} \beta_{A 2}\right) \\
V_{B}+\alpha_{B} \beta_{B 1}-\hat{y} t-P_{B 1}=V_{B}+\alpha_{B} \beta_{B 2}-(1-\hat{y}) t-P_{B 2} \\
\hat{y}=\frac{1}{2}+\frac{1}{2 t}\left(P_{B 2}-P_{B 1}+\alpha_{B} \beta_{B 1}-\alpha_{B} \beta_{B 2}\right)
\end{gathered}
$$

Las participaciones de mercado se muestran en la Figura 3. 
Figura 3. Participaciones de mercado sin empaquetamiento.

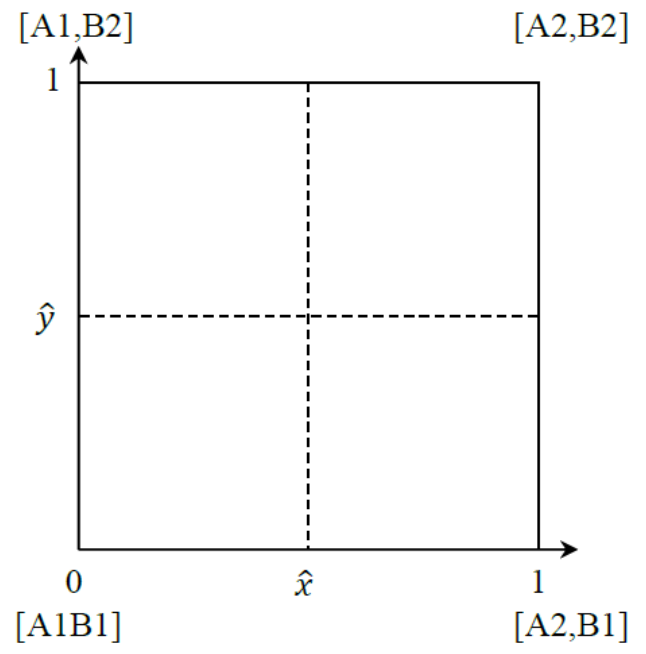

Fuente: Elaboración propia.

De acuerdo al supuesto de demanda unitaria, la demanda agregada para cada producto se deriva de la participación de mercado.

\section{Funciones de beneficios}

Las funciones de beneficios suman el ingreso menos los costos de inversión de las mejoras de calidad.

$\underline{\text { Firma } 1}$

$$
\pi_{1}\left(P_{A 1}, P_{B 1}, \beta_{A 1}, \beta_{B 1}\right)=\hat{x}\left[P_{A 1}-c_{A} \beta_{A 1}\right]+\hat{y}\left[P_{B 1}-c_{B} \beta_{B 1}\right]-b_{A} \beta_{A 1}^{2}-b_{B} \beta_{B 1}^{2}
$$

$\underline{\text { Firma } 2}$

$$
\pi_{A 2}\left(P_{A 2}, \beta_{A 2}\right)=(1-\hat{x})\left[P_{A 2}-c_{A} \beta_{A 2}\right]-b_{A} \beta_{A 2}^{2}
$$

$\underline{\text { Firma B2 }}$

$$
\pi_{B 2}\left(P_{B 2}, \beta_{B 2}\right)=(1-\hat{y})\left[P_{B 2}-c_{B} \beta_{B 2}\right]-b_{B} \beta_{B 2}^{2}
$$

\section{Excedente del consumidor}

Los consumidores evalúan los productos A y B de manera independiente.

$$
\begin{aligned}
C S=\int_{0}^{\hat{x}}\left[V_{A}+\alpha_{A} \beta_{A 1}\right. & \left.-x \theta t-P_{A 1}\right] d x+\int_{\hat{x}}^{1}\left[V_{A}+\alpha_{A} \beta_{A 2}-(1-x) \theta t-P_{A 2}\right] d x \\
& +\int_{0}^{\hat{y}}\left[V_{B}+\alpha_{B} \beta_{B 1}-y t-P_{B 1}\right] d y \\
& +\int_{\hat{y}}^{1}\left[V_{B}+\alpha_{B} \beta_{B 2}-(1-y) t-P_{B 2}\right] d y
\end{aligned}
$$

donde

$\hat{x}$ es la participación de firma 1 en mercado A.

$\hat{y}$ es la participación de firma 1 en mercado B. 


\section{(2) Estrategia de empaquetamiento puro.}

La firma 1 vende un producto empaquetado que contiene el producto A y el producto $\mathrm{B}$ al precio $\tilde{P}$. Los consumidores tienen solo dos elecciones, (I) y (IV). Los consumidores que son indiferentes entre [A1B1] y [A2, B2] enfrentan la siguiente condición:

$$
\begin{aligned}
V_{A}+V_{B}+\alpha_{A} \beta_{A 1}+\alpha_{B} \beta_{B 1}-x \theta t-y t-\tilde{P} \\
=V_{A}+V_{B}+\alpha_{A} \beta_{A 2}+\alpha_{B} \beta_{B 2}-(1-x) \theta t-(1-y) t-P_{A 2}-P_{B 2}
\end{aligned}
$$

Reordenando se tiene que:

$$
Y(x)=y=\frac{1+\theta}{2}-\theta x+\left(\frac{1}{2 t}\right)\left[P_{A 2}+P_{B 2}-\tilde{P}+\alpha_{A} \beta_{A 1}-\alpha_{A} \beta_{A 2}+\alpha_{B} \beta_{B 1}-\alpha_{B} \beta_{B 2}\right]
$$

Es posible escribir $y$ como una función de $x$ lo que se denota como $Y(x)$ y está representada por la línea punteada en la Figura 4. Los consumidores marginales están ubicados en esta línea. A un $x$ dado, el consumidor marginal que es indiferente entre el producto [A1B1] y [A2, B2] está ubicado en $(x, Y(x))$. Los consumidores en el área I compran el paquete [A1B1]. El resto en área II compran [A2, $\mathrm{B} 2$ ]. Debido al supuesto de demanda unitaria, la demanda por el producto A1 y B1 se deriva del área I y la demanda por el producto A2 y B2 se deriva del área II.

Figura 4. Participaciones de mercado con la estrategia de empaquetamiento puro.

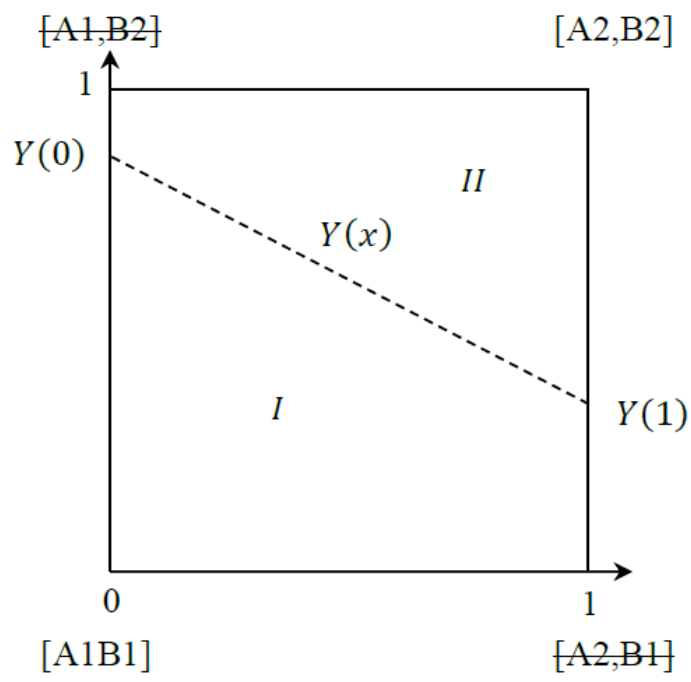

Fuente: Elaboración propia.

\section{Funciones de beneficios}

Las funciones de beneficios suman el ingreso menos los costos de inversión en mejoras de calidad. Esta sección enfatiza un rango de $\theta$ que hace la estrategia de empaquetamiento puro creíble. En este análisis del caso de empaquetamiento puro, $\theta$ no debería ser extremadamente alto para por garantizar que los beneficios de interrelacionar los mercados no son triviales.

$\underline{\text { Firma } 1}$

$$
\pi_{1}\left(\tilde{P}, \beta_{A 1}, \beta_{B 1}\right)=\frac{1}{2}[Y(0)+Y(1)]\left[\tilde{P}-c_{A} \beta_{A 1}-c_{B} \beta_{B 1}\right]-b_{A} \beta_{A 1}^{2}-b_{B} \beta_{B 1}^{2}
$$


Firma A2

$$
\pi_{A 2}\left(P_{A 2}, \beta_{A 2}\right)=\left\{1-\frac{1}{2}[Y(0)+Y(1)]\right\}\left[P_{A 2}-c_{A} \beta_{A 2}\right]-b_{A} \beta_{A 2}^{2}
$$

$\underline{\text { Firma B2 }}$

$$
\pi_{B 2}\left(P_{B 2}, \beta_{B 2}\right)=\left\{1-\frac{1}{2}[Y(0)+Y(1)\}\left[P_{B 2}-c_{B} \beta_{B 2}\right]-b_{B 2} \beta_{B 2}^{2}\right.
$$

\section{Excedente del consumidor}

$C S=\int_{0}^{1} \int_{0}^{Y(x)}\left[V_{A}+V_{B}+\alpha_{A} \beta_{A 1}+\alpha_{B} \beta_{B 1}-x \theta t-y t-\tilde{P}\right] d y d x+\int_{0}^{1} \int_{Y(x)}^{1}\left[V_{A}+V_{B}+\alpha_{A} \beta_{A 2}+\right.$

$\left.\alpha_{B} \beta_{B 2}-(1-x) \theta t-(1-y) t-P_{A 2}-P_{B 2}\right] d y d x$

donde $\tilde{P}$ es el precio del paquete puro [A1B1]; $P_{A 1 B 1}=\tilde{P}$.

\section{(3) Estrategia de empaquetamiento mixto.}

La firma 1 ofrece los productos separadamente a los precios $P_{A 1}$ y $P_{B 1}$. Adicionalmente, la firma 1 también ofrece un descuento de $\delta$ sobre el paquete [A1B1]. Los consumidores siguen teniendo a su disposición las cuatro elecciones, (I)-(IV). En la Figura 5, a un nivel dado de precios individuales y niveles de mejoras de calidad, $\hat{x}$ y $\hat{y}$ son las ubicaciones de los consumidores marginales en mercado $\mathrm{A}$ y mercado $\mathrm{B}$ respectivamente si el descuento del producto empaquetado es cero. Cuando la firma 1 ofrece el descuento sobre el producto empaquetado, su participación de mercado en ambos mercados aumenta en función de $\delta$. Este modelo está diseñado en un marco de dos mercados, similar a los de Gans y King (2006) y Matutes y Regibeau (1992).

Figura 5. Participaciones de mercado con la estrategia de empaquetamiento mixto.

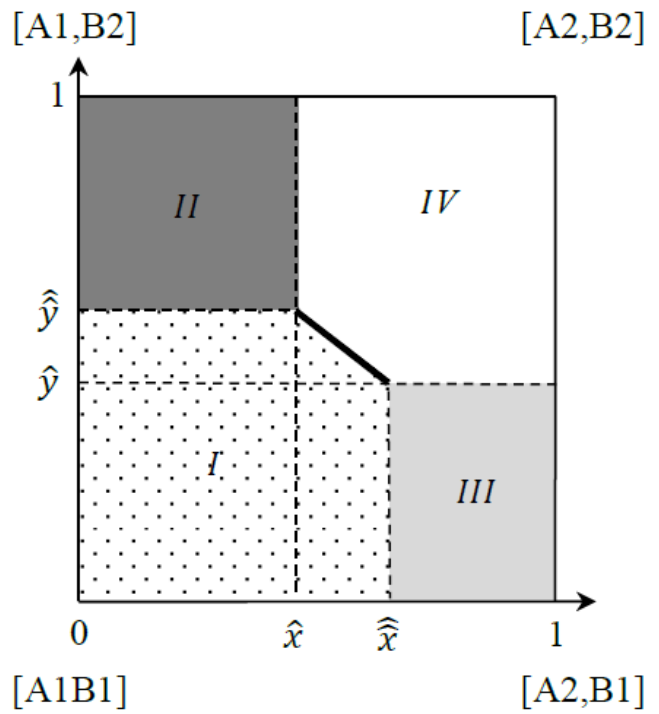

Fuente: Elaboración propia. 


$$
\begin{aligned}
& \hat{x}=\frac{1}{2}+\frac{1}{2 \theta t}\left(P_{A 2}-P_{A 1}+\alpha_{A} \beta_{A 1}-\alpha_{A} \beta_{A 2}\right) \\
& \hat{y}=\frac{1}{2}+\frac{1}{2 t}\left(P_{B 2}-P_{B 1}+\alpha_{B} \beta_{B 1}-\alpha_{B} \beta_{B 2}\right) \\
& \hat{\hat{x}}=\hat{x}+\frac{\delta}{2 \theta t} \\
& \hat{\hat{y}}=\hat{y}+\frac{\delta}{2 t}
\end{aligned}
$$

Los consumidores ubicados en el área I compran el paquete [A1B1] a la firma 1 a un precio descontado. Consumidores en las áreas II, III y IV compran los productos [A1, B2], [A2, B1] y [A2, B2] de firmas separadas respectivamente.

\section{Funciones de beneficios}

Las funciones de beneficios suman el ingreso menos los costos de inversión de las mejoras de calidad.

$\underline{\text { Firma } 1}$

$$
\pi_{1}\left(P_{A 1}, P_{B 1}, \delta, \beta_{A 1}, \beta_{B 1}\right)=D_{A 1}\left[P_{A 1}-c_{A} \beta_{A 1}\right]+D_{B 1}\left[P_{B 1}-c_{B} \beta_{B 1}\right]-D_{A 1 B 1} \delta-b_{A} \beta_{A 1}^{2}-b_{B} \beta_{A 2}^{2}
$$

$\underline{\text { Firma A2 }}$

$$
\pi_{A 2}\left(P_{A 2}, \beta_{A 2}\right)=D_{A 2}\left[P_{A 2}-c_{A} \beta_{A 2}\right]-b_{A} \beta_{A 2}^{2}
$$

\section{$\underline{\text { Firma B2 }}$}

$$
\pi_{B 2}\left(P_{B 2}, \beta_{B 2}\right)=D_{B 2}\left[P_{B 2}-c_{B} \beta_{B 2}\right]-b_{B} \beta_{B 2}^{2}
$$

$D_{A 1}$ es la demanda por el producto A1 de los consumidores que comprar el producto empaquetado [A1B1] o la combinación [A1, B2]. Así, $D_{A 1}$ es igual al total del área I y área II.

$$
D_{A 1}=\hat{x}+\frac{\delta}{2 \theta t} \hat{y}+\frac{1}{2}\left(\frac{\delta}{2 \theta t}\right)\left(\frac{\delta}{2 t}\right)
$$

$D_{B 1}$ es la demanda por el producto $\mathrm{B} 1$ de los consumidores que compran el producto empaquetado [A1B1] o la combinación [A2, B1]. Así, $D_{B 1}$ es igual al total de las áreas I y III.

$$
\begin{gathered}
D_{B 1}=\hat{y}+\frac{\delta}{2 t} \hat{x}+\frac{1}{2}\left(\frac{\delta}{2 \theta t}\right)\left(\frac{\delta}{2 t}\right) \\
D_{A 1 B 1}=\hat{x} \hat{y}+\hat{x}\left(\frac{\delta}{2 t}\right)+\hat{y}\left(\frac{\delta}{2 \theta t}\right)+\frac{1}{2}\left(\frac{\delta}{2 \theta t}\right)\left(\frac{\delta}{2 t}\right)
\end{gathered}
$$

$D_{A 2}$ es la demanda por el producto A2 de los consumidores que comprar la combinación [A2, $\mathrm{B} 1]$ o [A2, B2]. Así que, $D_{A 2}$ es igual a la suma del total de las áreas III y IV.

$$
D_{A 2}=1-\left\{\hat{x}+\frac{\delta}{2 \theta t} \hat{y}+\frac{1}{2}\left(\frac{\delta}{2 \theta t}\right)\left(\frac{\delta}{2 t}\right)\right\}
$$

$D_{B 2}$ es la demanda por el producto B2 de los consumidores que compran la combinación [A1, $\mathrm{B} 2]$ o [A2, B2]. Así que, $D_{B 2}$ es igual al total de las áreas II y IV. 


$$
D_{B 2}=1-\left\{\hat{y}+\frac{\delta}{2 t} \hat{x}+\frac{1}{2}\left(\frac{\delta}{2 \theta t}\right)\left(\frac{\delta}{2 t}\right)\right\}
$$

\section{Excedente del consumidor}

El excedente del consumidor después del empaquetamiento mixto es la suma del excedente del consumidor de las cuatro áreas. $Z(x)$ denota una función de $\mathrm{x}$ representada por la línea gruesa en Figura $5, x \in[\hat{x}, \hat{x}], Z(x) \in[\hat{y}, \hat{y}] . Z(x)=\hat{y}+\theta \hat{x}+\frac{\delta}{2 t}-\theta x$

$$
\begin{gathered}
C S=\int_{0}^{\hat{x}} \int_{0}^{\hat{y}}\left[V_{A}+V_{B}+\alpha_{A} \beta_{A 1}+\alpha_{B} \beta_{B 1}-x \theta t-y t-P_{A 1}-P_{B 1}-\delta\right] d y d x+\int_{\hat{x}}^{\hat{x}} \int_{0}^{Z(x)}\left[V_{A}+\right. \\
\left.V_{B}+\alpha_{A} \beta_{A 1}+\alpha_{B} \beta_{B 1}-x \theta t-y t-P_{A 1}-P_{B 1}-\delta\right] d y d x+\int_{0}^{\hat{x}} \int_{\hat{y}}^{1}\left[V_{A}+V_{B}+\alpha_{A} \beta_{A 1}+\alpha_{B} \beta_{B 2}-\right. \\
\left.x \theta t-(1-y) t-P_{A 1}-P_{B 2}\right] d y d x+\int_{\hat{x}}^{1} \int_{0}^{\hat{y}}\left[V_{A}+V_{B}+\alpha_{A} \beta_{A 2}+\alpha_{B} \beta_{B 1}-(1-x) \theta t-y t-P_{A 2}-\right. \\
\left.P_{B 1}\right] d y d x+\int_{\hat{x}}^{\hat{x}} \int_{Z(x)}^{1}\left[V_{A}+V_{B}+\alpha_{A} \beta_{A 2}+\alpha_{B} \beta_{B 2}-(1-x) \theta t-(1-y) t-P_{A 2}-P_{B 2}\right] d y d x+ \\
\int_{\hat{x}}^{1} \int_{\hat{y}}^{1}\left[V_{A}+V_{B}+\alpha_{A} \beta_{A 2}+\alpha_{B} \beta_{B 2}-(1-x) \theta t-(1-y) t-P_{A 2}-P_{B 2}\right] d y d x
\end{gathered}
$$

\section{Tiempo del juego}

Este modelo se reduce a un juego de tres etapas que se resuelve con inducción hacia atrás.

Etapa 1. La firma 1 decide si implementa las estrategias de empaquetamiento (empaquetamiento puro y empaquetamiento mixto) en vez de vender los productos A1 y B1 separadamente (sin empaquetamiento). Todas las firmas ya han entrado al mercado y, por lo tanto, el tema de entrada al mercado es omitido.

Etapa 2. Las firmas simultáneamente eligen sus niveles de mejoras de calidad.

Etapa 3. Las firmas fijan de manera simultánea los precios.

\section{Análisis.}

El resultado de la estrategia sin empaquetamiento se considera como el punto de referencia bajo el que se comparan los resultados de empaquetamiento puro y empaquetamiento mixto.

\subsection{Sin empaquetamiento.}

En la ausencia de empaquetamiento, la firma 1 compite con la firma A2 y la firma B2 en mercado A y mercado B separadamente. Los resultados de equilibrio se muestran a continuación.

\section{- Resultados de equilibrio del mercado}

Cuando no se permite que la firma 1 empaquete, los mercados son independientes los unos de los otros. Los consumidores toman sus elecciones en cada mercado de manera independiente.

Etapa 3. La firma soluciona el siguiente problema de maximización de beneficios:

$$
\underset{P_{A 1}, P_{B 1}}{\max } \pi_{1}
$$

Diferenciando la función de beneficios (3) con respecto a los precios asociados da como resultado: 


$$
\begin{aligned}
& \frac{\partial \pi_{1}}{\partial P_{A 1}}=\left\{\frac{1}{2}+\left(\frac{1}{2 \theta t}\right)\left[P_{A 2}-P_{A 1}+\alpha_{A} \beta_{A 1}-\alpha_{A} \beta_{A 2}\right]\right\}-\frac{1}{2 \theta t}\left(P_{A 1}-c_{A} \beta_{A 1}\right)=0 \\
& \frac{\partial \pi_{1}}{\partial P_{B 1}}=\left\{\frac{1}{2}+\left(\frac{1}{2 t}\right)\left[P_{B 2}-P_{B 1}+\alpha_{B} \beta_{B 1}-\alpha_{B} \beta_{B 2}\right]\right\}-\frac{1}{2 t}\left(P_{B 1}-c_{B} \beta_{B 1}\right)=0
\end{aligned}
$$

Análogamente, los problemas de las firmas monoproducto se muestran a continuación:

$$
\max _{P_{k 2}} \pi_{k 2}
$$

$k \in\{A, B\}$. Diferenciando las funciones de beneficios (4) y (5) con respecto a los precios asociados resulta:

$$
\begin{aligned}
& \frac{\partial \pi_{A 2}}{\partial P_{A 2}}=\left\{\frac{1}{2}-\left(\frac{1}{2 \theta t}\right)\left[P_{A 2}-P_{A 1}+\alpha_{A} \beta_{A 1}-\alpha_{A} \beta_{A 2}\right]\right\}-\frac{1}{2 \theta t}\left(P_{A 2}-c_{A} \beta_{A 2}\right)=0 \\
& \frac{\partial \pi_{B 2}}{\partial P_{B 2}}=\left\{\frac{1}{2}-\left(\frac{1}{2 t}\right)\left[P_{B 2}-P_{B 1}+\alpha_{B} \beta_{B 1}-\alpha_{B} \beta_{B 2}\right]\right\}-\frac{1}{2 t}\left(P_{B 2}-c_{B} \beta_{B 2}\right)=0
\end{aligned}
$$

Resolviendo (19) - (22) resultan las funciones de precios en la etapa 3 a un nivel dado de mejoras de calidad.

$$
\begin{aligned}
& P_{A 1}^{*}\left(\beta_{A 1}, \beta_{A 2}\right)=\theta t+\frac{2}{3} c_{A} \beta_{A 1}+\frac{1}{3} c_{A} \beta_{A 2}+\frac{1}{3}\left(\alpha_{A} \beta_{A 1}-\alpha_{A} \beta_{A 2}\right) \\
& P_{A 2}^{*}\left(\beta_{A 1}, \beta_{A 2}\right)=\theta t+\frac{1}{3} c_{A} \beta_{A 1}+\frac{2}{3} c_{A} \beta_{A 2}-\frac{1}{3}\left(\alpha_{A} \beta_{A 1}-\alpha_{A} \beta_{A 2}\right) \\
& P_{B 1}^{*}\left(\beta_{B 1}, \beta_{B 2}\right)=t+\frac{2}{3} c_{B} \beta_{B 1}+\frac{1}{3} c_{B} \beta_{B 2}+\frac{1}{3}\left(\alpha_{B} \beta_{B 1}-\alpha_{B} \beta_{B 2}\right) \\
& P_{B 2}^{*}\left(\beta_{B 1}, \beta_{B 2}\right)=t+\frac{1}{3} c_{B} \beta_{B 1}+\frac{2}{3} c_{B} \beta_{B 2}-\frac{1}{3}\left(\alpha_{B} \beta_{B 1}-\alpha_{B} \beta_{B 2}\right)
\end{aligned}
$$

Después de sustituir (23) - (26) en (3) - (5), las funciones de beneficios de las firmas en forma reducida se muestran a continuación.

$$
\begin{aligned}
& \pi_{1}\left(\beta_{A 1}, \beta_{A 2}, \beta_{B 1}, \beta_{B 2}\right)=\left[\frac{1}{2}-\frac{c_{A} \beta_{A 1}}{6 \theta t}+\frac{c_{A} \beta_{A 2}}{6 \theta t}+\frac{\alpha_{A} \beta_{A 1}-\alpha_{A} \beta_{A 2}}{6 \theta t}\right]\left[\theta t-\frac{1}{3} c_{A} \beta_{A 1}+\frac{1}{3} c_{A} \beta_{A 2}+\right. \\
& \left.\frac{1}{3}\left(\alpha_{A} \beta_{A 1}-\alpha_{A} \beta_{A 2}\right)\right]+\left[\frac{1}{2}-\frac{c_{B} \beta_{B 1}}{6 t}+\frac{c_{B} \beta_{B 2}}{6 t}+\frac{\alpha_{A} \beta_{A 1}-\alpha_{A} \beta_{A 2}}{6 t}\right]\left[t-\frac{1}{3} c_{B} \beta_{B 1}+\frac{1}{3} c_{B} \beta_{B 2}+\right. \\
& \left.\frac{1}{3}\left(\alpha_{B} \beta_{B 1}-\alpha_{B} \beta_{B 2}\right)\right]-b_{A} \beta_{A 1}^{2}-b_{B} \beta_{B 1}^{2} \\
& \pi_{A 2}\left(\beta_{A 1}, \beta_{A 2}, \beta_{B 1}, \beta_{B 2}\right)=\left[\frac{1}{2}+\frac{c_{A} \beta_{A 1}}{6 \theta t}+\frac{c_{A} \beta_{A 2}}{6 \theta t}+\frac{\alpha_{A} \beta_{A 1}-\alpha_{A} \beta_{A 2}}{6 \theta t}\right]\left[\theta t+\frac{1}{3} c_{A} \beta_{A 1}-\frac{1}{3} c_{A} \beta_{A 2}-\right. \\
& \left.\frac{1}{3}\left(\alpha_{A} \beta_{A 1}-\alpha_{A} \beta_{A 2}\right)\right]-b_{A} \beta_{A 2}^{2} \\
& \pi_{B 2}\left(\beta_{A 1}, \beta_{A 2}, \beta_{B 1}, \beta_{B 2}\right)=\left[\frac{1}{2}+\frac{c_{B} \beta_{B 1}}{6 t}+\frac{c_{B} \beta_{B 2}}{6 t}-\frac{\alpha_{A} \beta_{A 1}-\alpha_{A} \beta_{A 2}}{6 t}\right]\left[t+\frac{1}{3} c_{B} \beta_{B 1}-\frac{1}{3} c_{B} \beta_{B 2}-\right. \\
& \left.\frac{1}{3}\left(\alpha_{B} \beta_{B 1}-\alpha_{B} \beta_{B 2}\right)\right]-b_{B} \beta_{B 2}^{2}
\end{aligned}
$$

Etapa 2. La firma multiproducto y las firmas monoproductos maximizan sus propios beneficios como se muestra a continuación.

$$
\beta_{A 1, \beta_{B 1}}^{\max } \pi_{1}
$$




$$
\max _{\beta_{k 2}} \pi_{k 2}
$$

Es posible diferenciar (27) - (29) con respecto a los niveles de mejora de calidad $\left(\beta_{k i}\right) ; k \in$ $\{A, B\}, i \in\{1,2\}$. Después de solucionar las derivadas, los niveles de equilibrio de las mejoras de calidad se muestran a continuación.

$$
\begin{aligned}
& \beta_{A 1}^{*}=\beta_{A 2}^{*}=\frac{\alpha_{A}-c_{A}}{6 b_{A}} \\
& \beta_{B 1}^{*}=\beta_{B 2}^{*}=\frac{\alpha_{B}-c_{B}}{6 b_{B}}
\end{aligned}
$$

Sustituyendo (30) y (31) en las funciones de precios (23) - (26) da como resultado los precios de equilibrio que se muestran a continuación.

$$
\begin{aligned}
& P_{A 1}^{*}=P_{A 2}^{*}=\theta t+\frac{c_{A}\left(\alpha_{A}-c_{A}\right)}{6 b_{A}} \\
& P_{B 1}^{*}=P_{B 2}^{*}=t+\frac{c_{B}\left(\alpha_{B}-c_{B}\right)}{6 b_{B}}
\end{aligned}
$$

\section{Proposición 1}

Cuando no se permite el empaquetamiento, las firmas eligen precios y niveles de mejoras de calidad simétricos. Por lo tanto, las firmas tienen participaciones de mercado y beneficios iguales en cada mercado.

El resultado es consistente con el modelo estándar de Hotelling; las firmas eligen precios y niveles de mejoras de calidad simétricos en cada mercado porque la firma 1 compite con sus rivales en cada mercado separadamente. Los precios de equilibrio son determinados por los costos de degustación, los costos de mejora de calidad y la utilidad adicional de la mejora de calidad dentro del mismo mercado. Como una consecuencia del resultado simétrico en cada mercado separado, la firma multiproducto y los rivales monoproducto tienen participaciones de mercado y beneficios iguales en cada mercado.

\section{Observación 1}

Los costos de degustación tienen efectos positivos sobre los precios de equilibrio, pero no tienen efecto sobre los niveles de equilibrio de las mejoras de calidad.

$\underline{\text { Prueba }}$

De (30)-(33):

$$
\begin{gathered}
\frac{\partial P_{A 1}^{*}}{\partial t}=\frac{\partial P_{A 2}^{*}}{\partial t}=\theta>0 ; \frac{\partial P_{B 1}^{*}}{\partial t}=\frac{\partial P_{B 2}^{*}}{\partial t}=1>0 ; \\
\frac{\partial \beta_{A 1}^{*}}{\partial t}=\frac{\partial \beta_{A 2}^{*}}{\partial t}=0 ; \frac{\partial \beta_{B 1}^{*}}{\partial t}=\frac{\partial \beta_{B 2}^{*}}{\partial t}=0 .
\end{gathered}
$$

En cada mercado, las firmas eligen sus niveles de mejoras de calidad dependiendo únicamente de los costos relevantes y la utilidad adicional de las mejoras de calidad, pero independiente de los costos de degustación. En general, las firmas fijan precios para cubrir los costos marginales adicionales de las mejoras de calidad. En adición, ellas pueden aumentar los precios de acuerdo a los aumentos en los costos de degustación porque la sustituibilidad entre productos rivales decrece. Si la estructura de costos y utilidad adicional de las mejoras en calidad en los dos mercados son idénticas, el precio en el mercado menos competitivo es de hecho más alto. 


\section{Excedente del consumidor sin empaquetamiento}

Después de sustituir los resultados de equilibrio (30) - (33) en (6), el excedente del consumidor en el caso sin empaquetamiento es:

$$
C S=V_{A}+V_{B}+\frac{\left(\alpha_{A}-c_{A}\right)^{2}}{6 b_{A}}+\frac{\left(\alpha_{B}-c_{B}\right)^{2}}{6 b_{B}}-\frac{5 \theta t}{4}-\frac{5}{4} t
$$

\section{- Análisis de estática comparativa}

En el caso sin empaquetamiento, el equilibrio de los niveles de mejora en calidad $\left(\beta_{k i}\right)$ y precios $\left(P_{k i}\right)$ están determinados por los costos asociados y utilidad adicional en el mercado $k$.

\section{Mejoras en calidad}

De acuerdo a (30) - (31), el equilibrio de las mejoras de calidad es afectado por los costos y la adición de utilidad en la siguiente manera:

1) $\frac{\partial \beta_{k i}^{*}}{\partial b_{k}}=-\frac{\left(\alpha_{k}-c_{k}\right)}{6 b_{k}^{2}}<0$

Cuando los costos de inversión aumentan, es menos rentable para las firmas competir agresivamente en realizar mejoras de calidad. Por lo tanto, los niveles de equilibrio de las mejoras de calidad se reducen.

2) $\frac{\partial \beta_{k i}^{*}}{\partial c_{k}}=-\frac{1}{6 b_{k}}<0$

De igual modo, si los costos marginales adicionales se incrementan, las firmas tienen menos incentivos para invertir en mejoras en calidad.

3) $\frac{\partial \beta_{k i}^{*}}{\partial a_{k}}=\frac{1}{6 b_{k}}>0$

\section{Precio}

Considerando (32) - (33), los precios de equilibrio son claramente afectados por los costos y la utilidad adicional como se muestra a continuación.

1) $\frac{\partial \beta_{k i}^{*}}{\partial b_{k}}=-\frac{c_{k}\left(\alpha_{k}-c_{k}\right)}{6 b_{k}^{2}}<0$

Los precios se reducen con los costos de inversión. Cuando los costos de inversión se reducen, las firmas compiten aumentando los niveles de equilibrio de las mejoras de calidad para después aumentar los precios y poder así cubrir los costos incurridos por mejorar la calidad.

2) $\frac{\partial P_{k i}^{*}}{\partial c_{k}}=\frac{\left(\alpha_{k}-2 c_{k}\right)}{6 b_{k}}$

El efecto del costo marginal adicional sobre los precios es ambiguo. Los precios pueden caer con el costo marginal adicional si este costo es comparativamente alto $\left(c_{k}>\frac{\alpha_{k}}{2}\right)$. Bajo este supuesto, cuando los costos se incrementan, los niveles de mejoras en calidad se reducen y los costos marginales caen notablemente y, como consecuencia, también lo hacen los precios. De otro lado, cuando el costo es relativamente bajo $\left(c_{k}<\frac{\alpha_{k}}{2}\right)$, los precios pueden aumentar en función de los costos. Por ejemplo, cuando el costo por unidad de calidad se reduce, las firmas deciden aumentar sus niveles de mejoras en 
calidad. Sin embargo, el costo por unidad de calidad es tan bajo que los costos marginales adicionales se reducen. En conjunto, los precios caen.

3) $\frac{\partial P_{k i}^{*}}{\partial \alpha_{k}}=\frac{c_{k}}{6 b_{k}}>0$

Los precios aumentan con la utilidad adicional de las mejoras en calidad. Esto es porque este factor precisamente determina los niveles de equilibrio de las mejoras en calidad que las firmas tienen en cuenta en su decisión de precios.

\subsection{La estrategia de empaquetamiento puro.}

Cuando se permite el empaquetamiento puro, la firma 1 implementa esta estrategia que impacta los resultados del mercado como se presenta a continuación.

\section{- Resultados de equilibrio del mercado}

Etapa 3. La firma 1 solo vende su producto como un producto empaquetado, por lo tanto, los consumidores tienen solo dos elecciones, paquete [A1B1] y la combinación [A2, B2]. En esta etapa, la firma 1 soluciona el siguiente problema.

$$
\max _{\tilde{P}} \pi_{1}
$$

De (7), la condición de primer orden es:

$\frac{\partial \pi_{1}}{\partial \tilde{P}}=-\frac{1}{2 t}\left(\tilde{P}-c_{A} \beta_{A 1}-c_{B} \beta_{B 1}\right)+\frac{1}{2}+\left(\frac{1}{2 t}\right)\left[P_{A 2}+P_{B 2}-\tilde{P}+\alpha_{A} \beta_{A 1}-\alpha_{A} \beta_{A 2}+\alpha_{B} \beta_{B 1}-\alpha_{B} \beta_{B 2}\right]=0$

Análogamente, los problemas de las firmas monoproducto son:

$$
\max _{k 2} \pi_{k 2}
$$

donde $k \in\{A, B\}$. De (8) y (9), las condiciones de primer orden son como se presentan a continuación:

$$
\frac{\partial \pi_{A 2}}{\partial P_{A 2}}=-\frac{1}{2 t}\left(P_{A 2}-c_{A} \beta_{A 2}\right)+\frac{1}{2}+\left(\frac{1}{2 t}\right)\left[P_{A 2}+P_{B 2}-\tilde{P}+\alpha_{A} \beta_{A 1}-\alpha_{A} \beta_{A 2}+\alpha_{B} \beta_{B 1}-\alpha_{B} \beta_{B 2}\right]=0
$$

$\frac{\partial \pi_{B 2}}{\partial P_{B 2}}=-\frac{1}{2 t}\left(P_{B 2}-c_{B} \beta_{B 2}\right)+\frac{1}{2}+\left(\frac{1}{2 t}\right)\left[P_{A 2}+P_{B 2}-\tilde{P}+\alpha_{A} \beta_{A 1}-\alpha_{A} \beta_{A 2}+\alpha_{B} \beta_{B 1}-\alpha_{B} \beta_{B 2}\right]=0$

A cualquier combinación de mejoras en los niveles de calidad, los precios de equilibrio son:

$$
\begin{aligned}
& \tilde{P}^{*}\left(\beta_{A 1}, \beta_{A 2}, \beta_{B 1}, \beta_{B 2}\right)=\frac{5}{4} t+\frac{3}{4} c_{A} \beta_{A 1}+\frac{1}{4} c_{A} \beta_{A 2}+\frac{3}{4} c_{B} \beta_{B 1}+\frac{1}{4} c_{B} \beta_{B 2}+\frac{1}{4}\left[\alpha_{A} \beta_{A 1}-\alpha_{A} \beta_{A 2}+\right. \\
& \left.\alpha_{B} \beta_{B 1}-\alpha_{B} \beta_{B 2}\right] \\
& P_{A 2}^{*}\left(\beta_{A 1}, \beta_{A 2}, \beta_{B 1}, \beta_{B 2}\right)=\frac{3}{4} t+\frac{1}{4} c_{A} \beta_{A 1}+\frac{3}{4} c_{A} \beta_{A 2}+\frac{1}{4} c_{B} \beta_{B 1}-\frac{1}{4} c_{B} \beta_{B 2}-\frac{1}{4}\left[\alpha_{A} \beta_{A 1}-\alpha_{A} \beta_{A 2}+\right. \\
& \left.\alpha_{B} \beta_{B 1}-\alpha_{B} \beta_{B 2}\right] \\
& P_{B 2}^{*}\left(\beta_{A 1}, \beta_{A 2}, \beta_{B 1}, \beta_{B 2}\right)=\frac{3}{4} t+\frac{1}{4} c_{A} \beta_{A 1}-\frac{1}{4} c_{A} \beta_{A 2}+\frac{1}{4} c_{B} \beta_{B 1}+\frac{3}{4} c_{B} \beta_{B 2}-\frac{1}{4}\left[\alpha_{A} \beta_{A 1}-\alpha_{A} \beta_{A 2}+\right. \\
& \left.\alpha_{B} \beta_{B 1}-\alpha_{B} \beta_{B 2}\right]
\end{aligned}
$$

Sustituyendo (38) - (40) en (7) - (9), se obtiene lo siguiente: 
$\pi_{1}\left(\beta_{A 1}, \beta_{A 2}, \beta_{B 1}, \beta_{B 2}\right)=\left\{\left[\frac{5}{8}+\frac{1}{2 t}\left(\frac{1}{4} c_{A} \beta_{A 2}+\frac{1}{4} c_{B} \beta_{B 2}-\frac{1}{4} c_{A} \beta_{A 1}-\frac{1}{4} c_{B} \beta_{B 1}+\frac{1}{4}\left[\alpha_{A} \beta_{A 1}-\alpha_{A} \beta_{A 2}+\right.\right.\right.\right.$ $\left.\left.\left.\alpha_{B} \beta_{B 2}\right]\right)\right] *\left[\frac{5}{4} t-\frac{1}{4} c_{A} \beta_{A 1}+\frac{1}{4} c_{A} \beta_{A 2}-\frac{1}{4} c_{B} \beta_{B 1}+\frac{1}{4} c_{B} \beta_{B 2}+\frac{1}{4}\left[\alpha_{A} \beta_{A 1}-\alpha_{A} \beta_{A 2}+\alpha_{B} \beta_{B 1}-\right.\right.$ $\left.\left.\left.\alpha_{B} \beta_{B 2}\right]\right]\right\}-b_{A} \beta_{A 1}^{2}-b_{B} \beta_{B 1}^{2}$

$\pi_{A 2}\left(\beta_{A 1}, \beta_{A 2}, \beta_{B 1}, \beta_{B 2}\right)=\left\{\left[\frac{3}{8}-\frac{1}{2 t}\left(\frac{1}{4} c_{A} \beta_{A 2}+\frac{1}{4} c_{B} \beta_{B 2}-\frac{1}{4} c_{A} \beta_{A 1}-\frac{1}{4} c_{B} \beta_{B 1}+\frac{1}{4}\left[\alpha_{A} \beta_{A 1}-\alpha_{A} \beta_{A 2}+\right.\right.\right.\right.$ $\left.\left.\left.\alpha_{B} \beta_{B 1}-\alpha_{B} \beta_{B 2}\right]\right)\right] *\left[\frac{3}{4} t+\frac{1}{4} c_{A} \beta_{A 1}-\frac{1}{4} c_{A} \beta_{A 2}+\frac{1}{4} c_{B} \beta_{B 1}-\frac{1}{4} c_{B} \beta_{B 2}-\frac{1}{4}\left[\alpha_{A} \beta_{A 1}-\alpha_{A} \beta_{A 2}+\alpha_{B} \beta_{B 1}-\right.\right.$ $\left.\left.\left.\alpha_{B} \beta_{B 2}\right]\right]\right\}-b_{A} \beta_{A 2}^{2}$

$\pi_{B 2}\left(\beta_{A 1}, \beta_{A 2}, \beta_{B 1}, \beta_{B 2}\right)=\left\{\left[\frac{3}{8}-\frac{1}{2 t}\left(\frac{1}{4} c_{A} \beta_{A 2}+\frac{1}{4} c_{B} \beta_{B 2}-\frac{1}{4} c_{A} \beta_{A 1}-\frac{1}{4} c_{B} \beta_{B 1}+\frac{1}{4}\left[\alpha_{A} \beta_{A 1}-\alpha_{A} \beta_{A 2}+\right.\right.\right.\right.$ $\left.\left.\left.\alpha_{B} \beta_{B 1}-\alpha_{B} \beta_{B 2}\right]\right)\right] *\left[\frac{3}{4} t+\frac{1}{4} c_{A} \beta_{A 1}-\frac{1}{4} c_{A} \beta_{A 2}+\frac{1}{4} c_{B} \beta_{B 1}-\frac{1}{4} c_{B} \beta_{B 2}-\frac{1}{4}\left[\alpha_{A} \beta_{A 1}-\alpha_{A} \beta_{A 2}+\alpha_{B} \beta_{B 1}-\right.\right.$ $\left.\left.\left.\alpha_{B} \beta_{B 2}\right]\right]\right\}-b_{B} \beta_{B 2}^{2}$

Es posible diferenciar (41) - (43) con respecto a los niveles asociados de las mejoras en calidad $\left(\beta_{k i}\right) ; k \in\{A, B\}, i \in\{1,2\}$. Los resultados que se presentan a continuación satisfacen las condiciones de primer orden.

$$
\begin{aligned}
& \beta_{A 1}^{*}=\frac{\left(\alpha_{A}-c_{A}\right)\left[20 t b_{A} b_{B}-b_{B}\left(\alpha_{A}-c_{A}\right)^{2}-b_{A}\left(\alpha_{B}-c_{B}\right)^{2}\right]}{8 b_{A}\left[16 t b_{A} b_{B}-b_{B}\left(\alpha_{A}-c_{A}\right)^{2}-b_{A}\left(\alpha_{B}-c_{B}\right)^{2}\right]} \\
& \beta_{A 2}^{*}=\frac{\left(\alpha_{A}-c_{A}\right)\left[12 t b_{A} b_{B}-b_{B}\left(\alpha_{A}-c_{A}\right)^{2}-b_{A}\left(\alpha_{B}-c_{B}\right)^{2}\right]}{8 b_{A}\left[16 t b_{A} b_{B}-b_{B}\left(\alpha_{A}-c_{A}\right)^{2}-b_{A}\left(\alpha_{B}-c_{B}\right)^{2}\right]} \\
& \beta_{B 1}^{*}=\frac{\left(\alpha_{B}-c_{B}\right)\left[20 t b_{A} b_{B}-b_{B}\left(\alpha_{A}-c_{A}\right)^{2}-b_{A}\left(\alpha_{B}-c_{B}\right)^{2}\right]}{8 b_{B}\left[16 t b_{A} b_{B}-b_{B}\left(\alpha_{A}-c_{A}\right)^{2}-b_{A}\left(\alpha_{B}-c_{B}\right)^{2}\right]} \\
& \beta_{B 1}^{*}=\frac{\left(\alpha_{B}-c_{B}\right)\left[12 t b_{A} b_{B}-b_{B}\left(\alpha_{A}-c_{A}\right)^{2}-b_{A}\left(\alpha_{B}-c_{B}\right)^{2}\right]}{8 b_{A}\left[16 t b_{A} b_{B}-b_{B}\left(\alpha_{A}-c_{A}\right)^{2}-b_{A}\left(\alpha_{B}-c_{B}\right)^{2}\right]}
\end{aligned}
$$

Sustituyendo (44) - (47) en (38) - (40) se obtienen los precios de equilibrio del juego como se presenta a continuación:

$$
\begin{gathered}
\tilde{P}^{*}=\frac{5}{4} t+\left(\frac{1}{16}\right)\left[-\left(\frac{\left(\alpha_{A}-c_{A}\right)^{2}}{b_{A}}+\frac{\left(\alpha_{B}-c_{B}\right)^{2}}{b_{B}}\right)\right. \\
\left.+\left(\frac{20 t b_{A} b_{B}-b_{B}\left(\alpha_{A}-c_{A}\right)^{2}-b_{A}\left(\alpha_{B}-c_{B}\right)^{2}}{16 t b_{A} b_{B}-b_{B}\left(\alpha_{A}-c_{A}\right)^{2}-b_{A}\left(\alpha_{B}-c_{B}\right)^{2}}\right)\left(\frac{\alpha_{A}^{2}-c_{A}^{2}}{b_{A}}+\frac{\alpha_{B}^{2}-c_{B}^{2}}{b_{B}}\right)\right] \\
P_{A 2}^{*}=\frac{3}{4} t+\left(\frac{1}{16}\right)\left[\frac{3 c_{A}\left(\alpha_{A}-c_{A}\right)}{b_{A}}-\frac{c_{A}\left(\alpha_{A}-c_{A}\right)}{b_{A}}\left(\frac{20 t b_{A} b_{B}-b_{B}\left(\alpha_{A}-c_{A}\right)^{2}-b_{A}\left(\alpha_{B}-c_{B}\right)^{2}}{16 t b_{A} b_{B}-b_{B}\left(\alpha_{A}-c_{A}\right)^{2}-b_{A}\left(\alpha_{B}-c_{B}\right)^{2}}\right)-\right. \\
\left.\frac{4 \alpha_{A}\left(\alpha_{A}-c_{A}\right) t b_{B}+4\left(\alpha_{B}-c_{B}\right)^{2} t b_{A}}{16 t b_{A} b_{B}-b_{B}\left(\alpha_{A}-c_{A}\right)^{2}-b_{A}\left(\alpha_{B}-c_{B}\right)^{2}}\right] \\
P_{B 2}^{*}=\frac{3}{4} t+\left(\frac{1}{16}\right)\left[\frac{3 c_{B}\left(\alpha_{B}-c_{B}\right)}{b_{B}}-\frac{c_{B}\left(\alpha_{B}-c_{B}\right)}{b_{B}}\left(\frac{20 t b_{A} b_{B}-b_{B}\left(\alpha_{A}-c_{A}\right)^{2}-b_{A}\left(\alpha_{B}-c_{B}\right)^{2}}{16 t b_{A} b_{B}-b_{B}\left(\alpha_{A}-c_{A}\right)^{2}-b_{A}\left(\alpha_{B}-c_{B}\right)^{2}}\right)-\right. \\
\left.\frac{4 \alpha_{B}\left(\alpha_{B}-c_{B}\right) t b_{A}+4\left(\alpha_{A}-c_{A}\right)^{2} t b_{B}}{16 t b_{A} b_{B}-b_{B}\left(\alpha_{A}-c_{A}\right)^{2}-b_{A}\left(\alpha_{B}-c_{B}\right)^{2}}\right]
\end{gathered}
$$




\section{Proposición 2}

(i) Cuando la firma multiproducto emplea la estrategia de empaquetamiento puro, ésta elige un nivel más alto de mejoras en calidad que las firmas monoproducto, i.e. $\beta_{A 1}^{*}>\beta_{A 2}^{*}$ y $\beta_{B 1}^{*}>\beta_{B 2}^{*}$.

(ii) La firma multiproducto fija el precio del paquete por debajo del precio total de la combinación de producto de las firmas monoproducto cuando las mejoras en calidad involucran costos relativamente altos, pero resulta en menor utilidad adicional.

$\tilde{P}^{*}<(\geq) P_{A 2}^{*}+P_{B 2}^{*} \quad$ iff $\quad b_{B}\left(\alpha_{A}-c_{A}\right)^{2}+b_{A}\left(\alpha_{B}-c_{B}\right)^{2}+b_{B} c_{A}\left(\alpha_{A}-c_{A}\right)+b_{A} c_{B}\left(\alpha_{B}-c_{B}\right)<$
$(\geq) 4 t b_{A} b_{B}$

Una vez escogido el empaquetamiento puro, los consumidores tienen solo dos elecciones de productos, el paquete [A1B1] y la combinación [A2, B2]. La diferenciación entre estas dos elecciones está basada en el costo de degustación de los mercados menos competitivos. Los costos y la utilidad adicional de las mejoras en calidad en un mercado tienen algún tipo de efecto sobre los precios de equilibrio y los niveles de mejoras en calidad en el otro mercado. La firma multiproducto ciertamente elige niveles más altos de mejoras en calidad que sus rivales monoproducto. Esto es por incorporar la externalidad entre los dos mercados dentro de su problema de maximización de beneficios, pero las firmas monoproducto todavía maximizan sus propios beneficios de manera separada (sin cooperación). Choi (2004) y Krämer (2009) también reportaron resultados similares en su marco de empaquetamiento puro donde la firma multiproducto ata su producto de monopolio con su producto competitivo. Aunque en el presente estudio la firma multiproducto enfrenta competencia duopolista en ambos mercados, todavía emplea empaquetamiento puro para conseguir dominancia a través de su calidad superior.

La comparación de precios es ambigua. El precio del producto empaquetado de la firma multiproducto es más bajo que el precio de la combinación de producto de la firma monoproducto cuando los costos de mejoras en calidad, comparado con la utilidad adicional, son extremadamente altos. En este caso, los procesos de mejoras de calidad involucran costos altos de inversión y/o resultan en aumentos sustanciales de los costos marginales de producción, así que la firma multiproducto le da mayor énfasis al precio en comparación con la calidad debido a la ineficiencia en los costos de mejoras en calidad. Ésta ofrece niveles de mejoras en calidad más altos, y agresivamente fija precios más bajos que sus rivales para poder expandir su base de consumidores. En este modelo, los consumidores evalúan la elección de dos productos con respecto a la información perfecta sobre calidad y precio. Los consumidores pueden percibir el menor precio del paquete como un descuento indirecto. Esto es similar al resultado de Nalebuff (2004), donde no se les permite a las firmas modificar la calidad del producto en un contexto de empaquetamiento puro. Sin embargo, cuando los costos de mejora en calidad, comparados con los de la utilidad adicional, no son tan altos, la firma multiproducto se concentra en su inversión en mejoras de calidad y respectivamente fija el precio del paquete a un valor más alto que las firmas multiproducto.

\section{Observación 2}

Después del empaquetamiento puro, la competencia más intensa que se da en el mercado más competitivo $(\theta)$ ya no afecta los niveles de equilibrio de las mejoras en calidad y los precios.

Prueba

De acuerdo a (44) - (50):

$$
\frac{\partial \beta_{k i}^{*}}{\partial \theta}=0, \frac{\partial \tilde{P}^{*}}{\partial \theta}=0, \frac{\partial P_{A 2}^{*}}{\partial \theta}=0, \frac{\partial P_{B 2}^{*}}{\partial \theta}=0 ; k \in\{A, B\}, i \in\{1,2\}
$$

Después de empaquetamiento puro, solo [A1B1] y [A2, B2] quedan disponibles para el consumo. Desde el punto de vista de los consumidores, ellos pueden elegir entre [A1B1] o [A2, B2], y los 
productos en el mercado más competitivo se vuelven más diferenciados después de ser empaquetados con aquellos del mercado menos competitivo. Por lo tanto, los consumidores evalúan los dos productos disponibles principalmente basados en los costos de degustación del mercado menos competitivo $(t)$.

\section{Proposición 3}

Después del empaquetamiento, la firma multiproducto tiene mayores beneficios con una participación del mercado más alta que la firma monoproducto, i.e. $\pi_{1}^{*}>\pi_{A 2}^{*}+\pi_{B 2}^{*}$ y $M S_{1}>\frac{1}{2}>M S_{2}$.

Después de internalizar la externalidad entre los dos mercados interrelacionados, la firma multiproducto encuentra rentable ofrecer niveles de mejoras de calidad más altos para atraer un mayor número de consumidores y dominar el mercado a través de una participación de mercado más alta, lo que le genera mayores beneficios en comparación a los de sus rivales monoproducto.

\section{Excedente del consumidor con empaquetamiento puro}

El excedente del consumidor después del empaquetamiento puro es:

$C S=V_{A}+V_{B}-\frac{\theta^{2}}{6}-\frac{t}{2}+t Y^{0}\left(1-Y^{0}\right)-\theta t\left(1-Y^{0}\right)+\alpha_{A} \beta_{A 2}^{*}+\alpha_{B} \beta_{B 2}^{*}-\left(P_{A 2}^{*}+P_{B 2}^{*}\right)+\left(\frac{\theta}{2}-\right.$
$\left.Y^{0}\right)\left[\tilde{P}^{*}-\left(P_{A 2}^{*}+P_{B 2}^{*}\right)-\alpha_{A} \beta_{A 1}^{*}+\alpha_{A} \beta_{A 2}^{*}-\alpha_{B} \beta_{B 1}^{*}+\alpha_{B} \beta_{B 2}^{*}\right]$

donde $Y^{0}=Y(0)=\left(\frac{1+\theta}{2}\right)+\frac{2 t b_{A} b_{B}}{16 t b_{A} b_{B}-b_{B}\left(\alpha_{A}-c_{A}\right)^{2}-b_{A}\left(\alpha_{B}-c_{B}\right)^{2}}$

\section{- La estrategia de empaquetamiento puro vs. la estrategia sin empaquetamiento}

En la etapa 1, para enfatizar el efecto del empaquetamiento puro, los resultados de mercado de empaquetamiento puro son comparados con la situación de referencia, es decir, la situación sin empaquetamiento.

$$
\text { Denotando } \Omega=\frac{20 t b_{A} b_{B}-b_{B}\left(\alpha_{A}-c_{A}\right)^{2}-b_{A}\left(\alpha_{B}-c_{B}\right)^{2}}{16 t b_{A} b_{B}-b_{B}\left(\alpha_{A}-c_{A}\right)^{2}-b_{A}\left(\alpha_{B}-c_{B}\right)^{2}}, \mathbb{A}=\alpha_{A}-c_{A}>0, \mathbb{B}=\alpha_{B}-c_{B}>0
$$

\section{Proposición 4a}

Cuando la estrategia de empaquetamiento puro es implementada en vez de la estrategia sin empaquetamiento, los resultados de la firma multiproducto cambian como se presentan a continuación.

(i) En ambos mercados, la firma multiproducto reduce sus niveles de mejoras de calidad cuando las mejoras en calidad involucran costos relativamente altos, pero resulta en utilidad adicional comparativamente baja:

$$
\beta_{A 1 \text { Puro }}^{*}<\beta_{A 1 \text { No }}^{*}, \quad \beta_{B 1 \text { Puro }}^{*}<\beta_{B 1 \text { No }}^{*} \text { iff } b_{B} \mathbb{A}^{2}+b_{A} \mathbb{B}^{2}<4 t b_{A} b_{B}
$$

De manera opuesta, la firma multiproducto aumenta los niveles cuando el proceso de mejora en calidad es lo suficientemente eficiente en términos de costo y utilidad adicional:

$$
\beta_{A 1 \text { Puro }}^{*}>\beta_{A 1 \text { No }}^{*} \quad \beta_{B 1 \text { Puro }}^{*}>\beta_{B 1 \text { No }}^{*} \text { iff } 4 t b_{A} b_{B}<b_{B} \mathbb{A}^{2}+b_{A} \mathbb{B}^{2}<12 t b_{A} b_{B}
$$

(ii) La firma multiproducto aumenta el precio del paquete cuando los dos mercados interrelacionados son significativamente diferentes en términos de intensidad competitiva: 


$$
\begin{aligned}
& \tilde{P}_{\text {Puro }}^{*}>(\leq) P_{A 1 N o}^{*}+ P_{B 1 N o}^{*} \text { iff } \theta \\
&<(\geq) \frac{1}{4}+\frac{1}{48 t b_{A} b_{B}}\left\{(3 \Omega-3)\left[b_{B} \alpha_{A} \mathbb{A}+b_{A} \alpha_{B} \mathbb{B}\right]+(3 \Omega-5)\left[b_{B} c_{A} \mathbb{A}+b_{A} c_{B} \mathbb{B}\right]\right\}
\end{aligned}
$$

(iii) La participación de mercado de la firma multiproducto aumenta en ambos mercados, i.e. $M S_{1 \text { Puro }}>M S_{A 1 N o}=M S_{B 1 N o}$.

(iv) Los beneficios de la firma multiproducto aumentan, i.e. $\pi_{1 P u r o}^{*}>\pi_{1 N o}^{*}$. La estrategia de empaquetamiento puro domina la estrategia sin empaquetamiento para la firma multiproducto en muchas situaciones.

En esta sección se asume que $\theta$ no es extremadamente alta para lograr que la estrategia sea todavía creíble en el análisis de empaquetamiento puro.

Haciendo referencia a la estrategia sin empaquetamiento, la estrategia de empaquetamiento puro puede aumentar los beneficios de la firma multiproducto de diferentes formas. Consecuentemente, en la etapa 1 del juego, la firma 1 preferirá la estrategia de empaquetamiento puro a la estrategia sin empaquetamiento. Cuando los dos mercados están interrelacionados, las elecciones de producto son limitadas. La firma 1 toma esta oportunidad para reconsiderar sobre las mejoras en calidad y la fijación de precios teniendo en cuenta la externalidad dentro de su problema de maximización de beneficios. La firma 1 gastará menos en mejoras de calidad para ahorrar costos si el costo de inversión y el costo marginal adicional de las mejoras en calidad son muy altos en relación a una utilidad adicional significativamente baja. Puede que no sea rentable atraer consumidores mediante altas inversiones en mejoras en calidad con una tecnología ineficiente. Esto es porque los mercados parecen menos competitivos cuando solo dos opciones de las combinaciones de producto permanecen disponibles después del empaquetamiento puro. De otro modo, debido al efecto significativo de la externalidad, la estrategia de empaquetamiento puro puede estimular la inversión de la firma multiproducto si las mejoras de calidad no involucran altos costos de inversión y costos marginales adicionales pero la utilidad adicional es relativamente sustancial. Este estudio señala que los incentivos de la firma multiproducto para invertir en calidad dependen de la eficiencia de la aproximación de mejoras en calidad en términos de costos y utilidad adicional. Esto contrasta con el artículo de Choi (2004) que argumenta que la firma multiproducto ciertamente aumenta su inversión en reducción de costos después del empaquetamiento puro.

Los efectos del empaquetamiento puro sobre los precios son ambiguos. Dos efectos de empaquetamiento puro ocurren. Primero, el empaquetamiento puro reduce el número de elecciones de producto y reduce la fuerte competencia en el mercado A, así las firmas pueden cobrar precios más altos. Segundo, en respuesta al empaquetamiento puro, las firmas pueden cambiar sus niveles de mejoras en calidad, lo que directamente afecta los costos marginales y finalmente sus precios. Cuando los costos de degustación de los dos mercados son significativamente diferentes $(\theta$ es lo suficientemente bajo), el precio del paquete [A1B1] es más alto cuando se compara con la situación sin empaquetamiento. Cuando el mercado más competitivo está interrelacionado con el mercado menos competitivo, la reducción en la intensidad competitiva después del empaquetamiento puro beneficia la firma multiproducto de manera tan significativa que ésta puede aumentar el precio de su producto empaquetado. De otro modo, esta disminuirá su precio del paquete en el proceso de maximización de beneficios, lo cual internaliza la externalidad después del empaquetamiento puro. Con calidad más atractiva y precios razonables, la firma multiproducto puede aumentar su participación de mercado.

\section{Proposición 4b}

Cuando la firma multiproducto implementa la estrategia de empaquetamiento puro en vez de la estrategia sin empaquetamiento, las firmas monoproducto son afectadas como se muestra a continuación. 
(i) Las firmas monoproducto reducen sus niveles de mejoras en calidad en ambos mercados, i.e. $\beta_{A 2 \text { Puro }}^{*}<\beta_{A 2 N o}^{*} y \beta_{B 2 \text { Puro }}^{*}<\beta_{B 2 N o}^{*}$

(ii) La firma monoproducto en el mercado menos competitivo reduce su precio, i.e. $P_{B 2 \text { Puro }}^{*}<$ $P_{B 2 N o}^{*}$. Ambiguamente, la firma monoproducto en el mercado más competitivo aumenta su precio cuando los dos mercados interrelacionados son significativamente diferentes en intensidad competitiva:

$$
\begin{gathered}
P_{A 2 P \text { uro }}^{*}>(\leq) P_{A 2 N o}^{*} \text { iff } \\
\theta<(\geq) \frac{3}{4}-\frac{(3 \Omega-1) c_{A} \mathbb{A}\left(16 t b_{A} b_{B}-b_{B} \mathbb{A}^{2}-b_{A} \mathbb{B}^{2}\right)+12 \alpha_{A} \mathbb{A} t b_{A} b_{B}+12 \mathbb{B}^{2} t b_{A}^{2}}{48 t b_{A}\left(16 t b_{A} b_{B}-b_{B} \mathbb{A}^{2}-b_{A} \mathbb{B}^{2}\right)}
\end{gathered}
$$

(iii) Las participaciones de mercado de las firmas monoproducto caen en ambos mercados, i.e. $M S_{\text {A2Puro }}<M S_{A 2 N o}$ y $M S_{B 2 \text { Puro }}<M S_{B 2 N o}$.

(iv) En el mercado más competitivo, los beneficios de la firma monoproducto aumentan cuando los dos mercados interrelacionados son significativamente diferentes en intensidad competitiva:

$$
\begin{gathered}
\pi_{A 2 \text { Puro }}^{*}>(\leq) \pi_{A 2 N o}^{*} \text { iff } \\
\theta<(\geq)\left\{\frac{9}{16}+\frac{\mathbb{A}^{2}}{18 t b_{A}}\left[1-\frac{1}{2}\left[\frac{\left(12 t b_{A} b_{B}-b_{B} \mathbb{A}^{2}-b_{A} \mathbb{B}^{2}\right)}{\left(16 t b_{A} b_{B}-b_{B} \mathbb{A}^{2}-b_{A} \mathbb{B}^{2}\right)}\right]^{2}\right]-\frac{6}{16}\left[\frac{b_{B} \mathbb{A}^{2}+b_{A} \mathbb{B}^{2}}{\left(16 t b_{A} b_{B}-b_{B} \mathbb{A}^{2}-b_{A} \mathbb{B}^{2}\right)}\right]\right. \\
\left.+\frac{1}{16}\left[\frac{b_{B} \mathbb{A}^{2}+b_{A} \mathbb{B}^{2}}{\left(16 t b_{A} b_{B}-b_{B} \mathbb{A}^{2}-b_{A} \mathbb{B}^{2}\right)}\right]^{2}\right\}
\end{gathered}
$$

En el mercado menos competitivo, los beneficios de la firma monoproducto se reducen, i.e. $\pi_{B 2 \text { Puro }}^{*}<\pi_{B 2 N o}^{*}$

Cuando la firma multiproducto implementa el empaquetamiento puro, las firmas monoproducto deciden ahorrar en costos y reaccionan reduciendo sus niveles de mejoras de calidad, que terminan siendo más bajos que los de la firma 1. Esto es porque estas firmas de manera independiente consideran sus propios problemas de maximización de beneficios sin preocuparse por la externalidad. Este hallazgo confirma el resultado de Choi (2004) y Krämer (2009) que el empaquetamiento puro reduce el incentivo de los rivales de las firmas monoproducto para invertir, incluso en el entorno duopolístico con mayor intensidad competitiva.

De acuerdo a la proposición 2 y proposición 4a (i), con empaquetamiento puro, la firma 1 fortalece su posición en el mercado y lo demuestra invirtiendo más agresivamente en mejoras en calidad. La firma monoproducto en el mercado menos competitivo se ve adversamente afectada por la estrategia agresiva de empaquetamiento. Como un resultado, la firma B2 responde reduciendo el precio para maximizar sus beneficios en esta situación difícil. Finalmente, esta firma pierde su participación de mercado y beneficios. Sin embargo, los resultados de la firma monoproducto en el mercado más competitivo cambian de manera ambigua. Cuando los costos de degustación de los dos mercados son muy diferentes ( $\theta$ es muy bajo), la firma $\mathrm{A} 2$ se beneficia indirectamente del empaquetamiento puro de la firma 1. Cuando el mercado más competitivo está interrelacionado al mercado menos competitivo, la sustituibilidad entre elecciones de producto se reduce significativamente debido a las elecciones limitadas de producto que ahora enfrentan los consumidores. Por esta razón, la firma A2 puede aumentar su precio y sus beneficios crecen a pesar de que la participación de mercado se reduce. Por otro lado, interrelacionar los mercados lleva solo a una caída pequeña en el grado de competencia del mercado A, y consecuentemente la firma A2 tiene que reducir el precio debido a una reducción en su nivel de 
mejoras en calidad. En este caso, la firma A2, similar a la firma B2, gana una participación pequeña de mercado y disminuye sus beneficios comparado con la situación sin empaquetamiento.

\section{Proposición 4c}

Comparado al caso sin empaquetamiento, el efecto de la estrategia de empaquetamiento puro sobre el excedente del consumidor es ambiguo. El excedente del consumidor se reduce cuando los dos mercados interrelacionados son significativamente diferentes en intensidad competitiva:

$$
\begin{gathered}
C_{\text {Puro }}<(\geq) C S_{N o} \text { iff } \\
\theta(\theta+9)<(\geq) 9-9 \Omega+3 \Omega^{2}-\frac{1}{t}\left[\frac{\mathbb{A}^{2}}{b_{a}}+\frac{\mathbb{B}^{2}}{b_{B}}\right]\left[-2+\frac{15}{8} \Omega-\frac{3}{8} \Omega^{2}+\frac{(3 \Omega-6) t b_{A} b_{B}}{16 t b_{A} b_{B}-b_{B} \mathbb{A}^{2}-b_{A} \mathbb{B}^{2}}\right]
\end{gathered}
$$

Un cambio en el excedente del consumidor es ambiguo. El empaquetamiento puro reduce el excedente del consumidor cuando los costos de degustación de los dos mercados son significativamente diferentes ( $\theta$ es bajo). Las elecciones limitadas de producto generan distorsiones en las asignaciones de consumidores, lo que produce una desutilidad para el consumidor debido a la indisponibilidad de combinaciones de producto ideales para el consumidor. En adición, debido a una reducción en la intensidad competitiva producto del empaquetamiento puro, se genera una distorsión en precios, especialmente en el mercado más competitivo. Las distorsiones de precio y la asignación de consumidores afectan de manera adversa el bienestar del consumidor. Esto domina los beneficios de la decisión de la firma 1 sobre niveles de mejoras en calidad y el precio del producto empaquetado debido a la externalidad de la firma 1. De otro lado, el excedente del consumidor puede aumentar cuando los costos de degustación de los dos mercados no son significativamente diferentes $(\theta$ es lo suficientemente alto). Interrelacionar los dos mercados no lleva a distorsiones severas. Por lo tanto, el beneficio de la externalidad compensa la desutilidad y el efecto negativo de la distorsión en precios.

\subsection{La estrategia de empaquetamiento mixto.}

En adición al producto empaquetado, la firma 1 también ofrece sus productos separadamente de acuerdo con la estrategia de empaquetamiento mixto, que tiene efectos sobre los resultados del mercado tal y como se presenta a continuación.

\section{- Resultados del equilibrio de mercado}

Etapa 3. La firma multiproducto soluciona el siguiente problema.

$$
\underset{P_{A 1}, P_{B 1}, \delta^{\pi} \pi_{1}}{\max }
$$

Diferenciando (15) con respecto a los precios asociados y el descuento del paquete $(\delta)$ produce las siguientes condiciones de primer orden:

$$
\begin{gathered}
\frac{\partial \pi_{1}}{\partial P_{A 1}}=-\frac{1}{2 \theta t}\left(P_{A 1}-c_{A} \beta_{A 1}\right)-\frac{\delta}{4 \theta t^{2}}\left(P_{B 1}-c_{B} \beta_{B 1}\right)+\hat{x}+\frac{\delta \hat{y}}{\theta t}+\frac{3 \delta^{2}}{8 \theta t^{2}}=0 \\
\frac{\partial \pi_{1}}{\partial P_{B 1}}=-\frac{\delta}{4 \theta t^{2}}\left(P_{A 1}-c_{A} \beta_{A 1}\right)-\frac{1}{2 t}\left(P_{B 1}-c_{B} \beta_{B 1}\right)+\hat{y}+\frac{\delta \hat{x}}{t}+\frac{3 \delta^{2}}{8 \theta t^{2}}=0 \\
\frac{\partial \pi_{1}}{\partial \delta}=\left\{\left(\frac{\hat{y}}{2 \theta t}+\frac{\delta}{4 \theta t^{2}}\right)\left(P_{A 1}-c_{A} \beta_{A 1}\right)\left(\frac{\hat{x}}{2 t}+\frac{\delta}{4 \theta t^{2}}\right)\left(P_{B 1}-c_{B} \beta_{B 1}\right)-\hat{x} \hat{y}-\frac{\delta \hat{x}}{t}-\frac{\delta \hat{y}}{\theta t}-\frac{3 \delta^{2}}{8 \theta t^{2}}\right\}=0
\end{gathered}
$$

De manera análoga, el problema de maximización para las firmas monoproducto es: 


$$
\max _{\beta_{k 2}} \pi_{k 2}
$$

$k \in\{A, B\}$. Las condiciones de primer obtenidas de diferenciar (16), (17) con respecto a los precios asociados se muestran a continuación.

$$
\begin{aligned}
& \frac{\partial \pi_{A 2}}{\partial P_{A 2}}=-\frac{1}{2 \theta t}\left(P_{A 2}-c_{A} \beta_{A 2}\right)+1-\hat{x}-\frac{\delta \hat{y}}{2 \theta t}-\frac{\delta^{2}}{8 \theta t^{2}}=0 \\
& \frac{\partial \pi_{B 2}}{\partial P_{B 2}}=-\frac{1}{2 t}\left(P_{B 2}-c_{B} \beta_{B 2}\right)+1-\hat{y}-\frac{\delta \hat{x}}{2 t}-\frac{\delta^{2}}{8 \theta t^{2}}=0
\end{aligned}
$$

donde

$$
\begin{aligned}
& \hat{x}=\frac{1}{2}+\frac{1}{2 \theta t}\left(P_{A 2}-P_{A 1}+\alpha_{A} \beta_{A 1}-\alpha_{A} \beta_{A 2}\right) \\
& \hat{y}=\frac{1}{2}+\frac{1}{2 t}\left(P_{B 2}-P_{B 1}+\alpha_{B} \beta_{B 1}-\alpha_{B} \beta_{B 2}\right)
\end{aligned}
$$

La función del descuento $(\delta)$ de equilibrio por parte de la firma 1 no puede ser explícitamente expresada en forma cerrada. Sin embargo, después de considerar (54) en el vecindario de $\delta=0$, se encuentra que:

$$
\left.\frac{\partial \pi_{1}}{\partial \delta}\right|_{\delta=0}=\left(\frac{\hat{y}}{2 \theta t}\right)\left(P_{A 1}-c_{A} \beta_{A 1}\right)+\left(\frac{\hat{x}}{2 t}\right)\left(P_{B 1}-c_{B} \beta_{B 1}\right)-\hat{x} \hat{y}
$$

Si $\delta=0$, la competencia por empaquetamiento mixto se convierte al caso sin empaquetamiento en el cual las firmas eligen niveles de mejoras en calidad simétricos y precios en equilibrio.

Por lo tanto,

$$
\left.\frac{\partial \pi_{1}}{\partial \delta}\right|_{\delta=0}=\frac{1}{4}>0
$$

Como un resultado, si el empaquetamiento mixto es permitido, la firma 1 tiene un incentivo para ofrecer un descuento sobre el producto empaquetado para poder aumentar sus beneficios. Así, en equilibrio $\delta^{*}>0$.

Debido al hecho que en el descuento del paquete en equilibrio no puede ser expresado en forma cerrada, se pueden escribir los precios de equilibrio como una función de los niveles de mejoras en calidad y el descuento del paquete en equilibrio $\left(\delta^{*}\right)$. Después de solucionar $(52)-(56)$, los precios de equilibrio a cualquier nivel dado de niveles de mejoras en calidad se pueden expresar tal y como se presenta a continuación.

$$
\begin{aligned}
& P_{A 1}^{*}\left(\beta_{A 1}, \beta_{A 2}, \beta_{B 1}, \beta_{B 2}\right)=\left(\frac{144 \theta t^{2}}{144 \theta^{2} t^{4}-76 \theta t^{2} \delta^{* 2}+\delta^{* 4}}\right)\left[\theta\left(\theta t^{3}-\frac{\delta^{* 2} t}{3}\right)+\frac{\delta^{*} \theta t^{2}}{6}+\frac{\delta^{* 5}}{288 \theta t}-\frac{2}{9} \delta^{* 3}+\right. \\
& \left(\frac{960 \theta^{2} t^{4}-40 \theta t^{2} \delta^{* 2}+\delta^{* 4}}{144 \theta t^{2}}\right) c_{A} \beta_{A 1}+\left(\frac{40 \theta t^{2}-3 \delta^{* 2}}{12}\right) c_{A} \beta_{A 2}-\theta\left(\frac{2 \delta \theta t^{2}+\delta^{* 3}}{36 \theta t}\right) c_{B} \beta_{B 1}+\theta\left(\frac{2 \delta \theta t^{2}+\delta^{* 3}}{36 \theta t}\right) c_{B} \beta_{B 2}+ \\
& \left.\left(\frac{4 \theta t^{2}-3 \delta^{* 2}}{12}\right)\left(\alpha_{A} \beta_{A 1}-\alpha_{A} \beta_{A 2}\right)+\theta\left(\frac{2 \delta \theta t^{2}+\delta^{* 3}}{36 \theta t}\right)\left(\alpha_{B} \beta_{B 1}-\alpha_{B} \beta_{B 2}\right)\right]
\end{aligned}
$$




$$
\begin{aligned}
& P_{B 1}^{*}\left(\beta_{A 1}, \beta_{A 2}, \beta_{B 1}, \beta_{B 2}\right)=\left(\frac{144 \theta t^{2}}{144 \theta^{2} t^{4}-76 \theta t^{2} \delta^{* 2}+\delta^{* 4}}\right)\left[\left(\theta t^{3}-\frac{\delta^{* 2} t}{3}\right)+\frac{\delta^{*} \theta t^{2}}{6}+\frac{\delta^{* 5}}{288 \theta t}-\frac{2}{9} \delta^{* 3}-\right. \\
& \left(\frac{2 \delta^{*} \theta t^{2}+\delta^{* 3}}{36 \theta t}\right) c_{A} \beta_{A 1}+\left(\frac{2 \delta^{*} \theta t^{2}+\delta^{* 3}}{36 \theta t}\right) c_{A} \beta_{A 2}+\left(\frac{96 \theta^{2} t^{4}-40 \theta t^{2} \delta^{* 2}+\delta^{* 4}}{144 \theta t^{2}}\right) c_{B} \beta_{B 1}+\left(\frac{4 \theta t^{2}-3 \delta^{* 2}}{12}\right) c_{B} \beta_{B 2}+ \\
& \left.\left(\frac{2 \delta \theta t^{2}+\delta^{* 3}}{36 \theta t}\right)\left(\alpha_{A} \beta_{A 1}-\alpha_{A} \beta_{A 2}\right)+\left(\frac{4 \theta t^{2}-3 \delta^{* 2}}{12}\right)\left(\alpha_{B} \beta_{B 1}-\alpha_{B} \beta_{B 2}\right)\right]
\end{aligned}
$$

$$
\begin{aligned}
& P_{A 2}^{*}\left(\beta_{A 1}, \beta_{A 2}, \beta_{B 1}, \beta_{B 2}\right)=\frac{\delta^{*}}{6 t}\left(\frac{144 \theta t^{2}}{144 \theta^{2} t^{4}-76 \theta t^{2} \delta^{* 2}+\delta^{* 4}}\right)\left[\left(\theta t^{3}-\frac{\delta^{* 2} t}{3}\right)+\frac{\delta^{*} \theta t^{2}}{6}+\frac{\delta^{* 5}}{288 \theta t}-\frac{2}{9} \delta^{* 3}-\right. \\
& \left(\frac{2 \delta^{*} \theta t^{2}+\delta^{* 3}}{36 \theta t}\right) c_{A} \beta_{A 1}+\left(\frac{2 \delta^{*} \theta t^{2}+\delta^{* 3}}{36 \theta t}\right) c_{A} \beta_{A 2}+\left(\frac{96 \theta^{2} t^{4}-40 \theta t^{2} \delta^{* 2}+\delta^{* 4}}{144 \theta t^{2}}\right) c_{B} \beta_{B 1}+\left(\frac{4 \theta t^{2}-3 \delta^{* 2}}{12}\right) c_{B} \beta_{B 2}+ \\
& \left.\left(\frac{2 \delta \theta t^{2}+\delta^{* 3}}{36 \theta t}\right)\left(\alpha_{A} \beta_{A 1}-\alpha_{A} \beta_{A 2}\right)+\left(\frac{4 \theta t^{2}-3 \delta^{* 2}}{12}\right)\left(\alpha_{B} \beta_{B 1}-\alpha_{B} \beta_{B 2}\right)\right]+\theta t+\frac{\delta^{* 2}}{12 t}+\frac{1}{3} c_{A} \beta_{A 1}+\frac{2}{3} c_{A} \beta_{A 2}+ \\
& \frac{\delta^{*}}{6 t} c_{B} \beta_{B 1}-\frac{1}{3}\left(\alpha_{A} \beta_{A 1}-\alpha_{A} \beta_{A 2}\right)
\end{aligned}
$$

$$
\begin{aligned}
& P_{B 2}^{*}\left(\beta_{A 1}, \beta_{A 2}, \beta_{B 1}, \beta_{B 2}\right)=\frac{\delta^{*}}{6 \theta t}\left(\frac{144 \theta t^{2}}{144 \theta^{2} t^{4}-76 \theta t^{2} \delta^{* 2}+\delta^{* 4}}\right)\left[\theta\left(\theta t^{3}-\frac{\delta^{* 2} t}{3}\right)+\frac{\delta^{*} \theta t^{2}}{6}+\frac{\delta^{* 5}}{288 \theta t}-\frac{2}{9} \delta^{* 3}+\right. \\
& \left(\frac{96 \theta^{2} t^{4}-40 \theta t^{2} \delta^{* 2}+\delta^{* 4}}{144 \theta t^{2}}\right) c_{B} \beta_{A 1}+\left(\frac{4 \theta t^{2}-3 \delta^{* 2}}{12}\right) c_{B} \beta_{A 2}-\theta\left(\frac{2 \delta \theta t^{2}+\delta^{* 3}}{36 \theta t}\right) c_{B} \beta_{B 1}+\left(\frac{4 \theta t^{2}-3 \delta^{* 2}}{12}\right)\left(\alpha_{A} \beta_{A 1}-\right. \\
& \left.\left.\alpha_{A} \beta_{A 2}\right)+\theta\left(\frac{2 \delta \theta t^{2}+\delta^{* 3}}{36 \theta t}\right)\left(\alpha_{B} \beta_{B 1}-\alpha_{B} \beta_{B 2}\right)\right]+t+\frac{\delta^{* 2}}{12 \theta t}+\frac{\delta^{*}}{6 \theta t} c_{A} \beta_{A 1}+\frac{1}{3} c_{B} \beta_{B 1}+\frac{2}{3} c_{B} \beta_{B 2}- \\
& \frac{1}{3}\left(\alpha_{B} \beta_{B 1}-\alpha_{B} \beta_{B 2}\right)
\end{aligned}
$$

Etapa 2. Las firmas eligen los niveles de mejoras en calidad simultáneamente. El problema de la firma multiproducto y de las firmas monoproducto son como siguen.

$$
\begin{aligned}
& \max _{\beta_{A 1}, \beta_{B 1}} \pi_{1} \\
& \max _{\beta_{k 2}} \pi_{k 2}
\end{aligned}
$$

Es posible diferenciar las funciones de beneficios en forma reducida con respecto a los niveles asociados de mejoras en calidad $\left(\beta_{k i}\right): k \in\{A, B\}, i \in\{1,2\}$. Adicionalmente, se puede sustituir (55) y (56) dentro de las derivadas para obtener:

$$
\begin{aligned}
& \frac{\partial \pi_{1}}{\partial \beta_{A 1}}=\left[1-\frac{1}{2 \theta t}\left(P_{A 2}-c_{A} \beta_{A 2}\right)\right]\left(\frac{\partial P_{A 2}}{\partial \beta_{A 1}}+\alpha_{A}-c_{A}\right)+\left[1-\frac{1}{2 t}\left(P_{B 2}-c_{B} \beta_{B 2}\right)\right] \frac{\partial P_{B 2}}{\partial \beta_{A 1}}-2 b_{A} \beta_{A 1}=0 \\
& \frac{\partial \pi_{1}}{\partial \beta_{B 1}}=\left[1-\frac{1}{2 \theta t}\left(P_{A 2}-c_{A} \beta_{A 2}\right)\right] \frac{\partial P_{A 2}}{\partial \beta_{B 1}}+\left[1-\frac{1}{2 t}\left(P_{B 2}-c_{B} \beta_{B 2}\right)\right]\left(\frac{\partial P_{B 2}}{\partial \beta_{A 1}}+\alpha_{B}-c_{B}\right)-2 b_{B} \beta_{B 1}=0 \\
& \frac{\partial \pi_{A 2}}{\partial \beta_{A 2}}=\frac{1}{2 \theta t}\left(P_{A 2}-c_{A} \beta_{A 2}\right)\left[\frac{\partial P_{A 1}}{\partial \beta_{A 2}}+\alpha_{A}-c_{A}-\frac{\delta}{2 t} \frac{\partial P_{B 2}}{\partial \beta_{A 2}}-\left(\hat{y}+\frac{\delta}{2 t}\right) \frac{\partial \delta}{\partial \beta_{A 2}}\right]-2 b_{A} \beta_{A 2}=0 \\
& \frac{\partial \pi_{B 2}}{\partial \beta_{B 2}}=\frac{1}{2 t}\left(P_{B 2}-c_{B} \beta_{B 2}\right)\left[\frac{\partial P_{B 1}}{\partial \beta_{B 2}}+\alpha_{B}-c_{B}-\frac{\delta}{2 \theta t} \frac{\partial P_{A 2}}{\partial \beta_{B 2}}+\frac{\delta}{2 \theta t} \frac{\partial P_{A 1}}{\partial \beta_{B 2}}-\left(\hat{x}+\frac{\delta}{2 \theta t}\right) \frac{\partial \delta}{\partial \beta_{B 2}}\right]-2 b_{B} \beta_{B 2}=0
\end{aligned}
$$

\section{Proposición 5}

(i) La firma multiproducto ofrece un descuento sobre el paquete $\left(\delta^{*}>0\right)$ con la estrategia de empaquetamiento mixto y fija precios individuales más altos que los fijados por los rivales monoproducto, i.e. $P_{A 1}^{*}>P_{A 2}^{*}$ y $P_{B 1}^{*}>P_{B 2}^{*}$. 
(ii) En el mercado más competitivo, los niveles de mejora en calidad de la firma multiproducto son más altos que los de su contraparte monoproducto.

El resultado de mejoras en calidad en el mercado menos competitiva es ambiguo de acuerdo a $\theta$. Sin embargo, si los dos mercados son idénticos en estructura de costos y utilidad adicional de mejoras en calidad y los costos de degustación no son tan diferentes ( $\theta$ es lo suficientemente alto), el resultado es similar a los del mercado más competitivo.

El caso sin empaquetamiento puede ser visto como el caso de empaquetamiento mixto con $\delta=$ 0 . La firma multiproducto ofrece un descuento sobre el paquete $\left(\delta^{*}\right)$. Aunque los precios de la firma multiproducto son más altos que los precios de sus rivales, sus productos son todavía atractivos porque puede ofrecer un descuento como una herramienta de discriminación de precios bajo la estrategia de empaquetamiento mixto. La firma multiproducto recompensa a los consumidores al comprar sus paquetes con un descuento. Adicionalmente, en el mercado más competitivo, realiza mayores inversiones en mejoras en calidad para atraer más consumidores, especialmente esos que no compran su paquete, pero compran o el producto A1 o el producto B1. Las firmas monoproducto no pueden discriminar consumidores mediante un descuento sobre el paquete. En respuesta a los precios descontados de la firma multiproducto, las firmas monoproducto (sin cooperación) tienen que atraer consumidores fijando menores precios, lo que afecta todos los consumidores en el mismo modo. Esta reacción se encuentra comúnmente como complementos estratégicos en un juego de precios. Así que, en el mercado más competitivo, la firma monoproducto ciertamente reduce sus niveles de mejoras en calidad para ahorrar costos. Estos resultados de mejoras en calidad también se encuentran en el mercado menos competitivo si los costos de degustación de los dos mercados no son tan diferentes $(\theta$ es lo suficientemente alto). Aunque la firma multiproducto renuncia a algunos beneficios debido al descuento del paquete, puede después de todo estimular sus beneficios. Esto es parcialmente debido a sus precios individuales más altos que los de las firmas monoproducto.

La discriminación de calidad no es práctica en esta configuración. La firma multiproducto ofrece los mismos niveles de mejoras en calidad a todos los consumidores. Suponiendo que los costos de degustación de los dos mercados no son tan diferentes ( $\theta$ es lo suficientemente alto), la firma multiproducto decide fijar niveles de mejoras en calidad que las firmas monoproducto para poder atraer los consumidores que confrontan sus precios individuales más altos. Similarmente, con la estrategia de empaquetamiento puro, como se estableció en proposición 2, la firma multiproducto también elige invertir en mejoras en calidad de manera más intensa que sus rivales monoproducto.

\section{Proposición 6}

(i) Después del empaquetamiento mixto, la firma multiproducto tiene beneficios más altos que las firmas monoproducto, i.e. $\pi_{1}^{*}>\pi_{A 2}^{*}+\pi_{B 2}^{*}$.

(ii) Las participaciones de equilibrio de mercado son ambiguas.

En el mercado más competitivo es posible que la firma multiproducto tenga participaciones de mercado mayores que la firma monoproducto.

El resultado en el mercado menos competitivo es ambiguo de acuerdo a $\theta$. Sin embargo, si los dos mercados son idénticos en estructura de costos y utilidad adicional de las mejoras en calidad y los costos de degustación en los dos mercados no son tan diferentes ( $\theta$ es lo suficientemente alto), el resultado es similar al que se presenta en el mercado más competitivo.

La firma multiproducto puede persuadir algunos consumidores para que se cambien a su producto por medio de estrategias de empaquetamiento mixto. Comparado al mercado menos competitivo, es más probable que la firma multiproducto quite algo de participación de mercado a su competidor monoproducto y se vuelva dominante en el mercado más competitivo con costos de degustación menores. El resultado en el mercado menos competitivo es ambiguo. Sin embargo, si los dos mercados 
son idénticos en estructura de costos y la utilidad adicional en mejoras en calidad y los costos de degustación en los dos mercados no son tan diferentes ( $\theta$ es lo suficientemente alto), es posible encontrar esta participación de mercado en el mercado menos competitivo. Adicionalmente, es cierto que la suma de los beneficios de las firmas monoproducto es menor que los beneficios de la firma multiproducto. La estrategia de empaquetamiento mixto pone las firmas monoproducto en desventaja.

En adición a la estrategia de empaquetamiento, la firma multiproducto puede obtener participaciones de mercado más altas que sus rivales después de adoptar la estrategia de empaquetamiento mixto. Tanto el empaquetamiento puro como el empaquetamiento mixto puede incrementar los beneficios de la firma multiproducto y obtener beneficios más altos que los de sus rivales.

\section{- La estrategia de empaquetamiento puro vs. la estrategia sin empaquetamiento}

Los efectos de la estrategia de empaquetamiento sobre los resultados del mercado son discutidos en forma de una comparación entre los resultados de empaquetamiento mixto y la referencia sin empaquetamiento.

\section{Proposición 7a}

Después de la implementación de la estrategia de empaquetamiento mixto y no la sin empaquetamiento, los beneficios de la firma multiproducto aumentan mientras que los beneficios de las firmas monoproducto se reducen en ambos mercados, $\pi_{1 \text { Mixto }}^{*}>\pi_{1 N o}^{*}, \pi_{A 2 \text { Mixto }}^{*}>\pi_{A 2 \text { No }}^{*} y$ $\pi_{B 2 M i x t o}^{*}>\pi_{B 2 N o}^{*}$. La estrategia de empaquetamiento puro domina la estrategia de no empaquetamiento para la firma multiproducto.

En la etapa 1 del juego, la firma multiproducto tiene incentivos para emplear la estrategia de empaquetamiento mixto en vez de la sin empaquetamiento porque puede aumentar el beneficio después del empaquetamiento mixto. El descuento sobre el paquete puede ser visto como una herramienta para que la firma multiproducto persuada a los consumidores que originalmente compraran otras combinaciones de producto para que elijan su paquete [A1B1].

\section{Proposición $7 b$}

Cuando la firma multiproducto emplea la estrategia de empaquetamiento mixto en vez de la estrategia sin empaquetamiento, los precios y las mejoras en calidad cambian como se presenta a continuación.

(i) La firma multiproducto aumenta sus precios individuales, mientras que las firmas monoproducto reducen sus precios en ambos mercados, i.e. $P_{A 1 \text { Mixto }}^{*}>P_{A 1 N o}^{*}, P_{B 1 \text { Mixto }}^{*}>$ $P_{B 1 \text { No }}^{*}, P_{A 2 M i x t o}^{*}<P_{A 2 N o}^{*} y P_{B 2 M i x t o}^{*}>P_{B 2 N o}^{*}$.

(ii) En el mercado más competitivo, la firma multiproducto aumenta sus niveles de mejoras en calidad, pero la firma monoproducto reduce sus niveles de mejoras en calidad, i.e. $\beta_{A 1 \text { Mixto }}^{*}>$ $\beta_{\text {A1No }}^{*} y \beta_{\text {A2Mixto }}^{*}<\beta_{\text {A2No }}^{*}$

Un cambio en las mejoras de calidad en el mercado menos competitivo es ambiguo. Sin embargo, si los dos mercados son idénticos en estructura de costos y utilidad adicional de las mejoras de calidad $y$ los costos de degustación de los dos mercados no son tan diferentes ( $\theta$ es lo suficientemente alto), la firma multiproducto aumenta sus niveles de mejoras de calidad, pero la firma monoproducto reduce sus niveles de mejoras de calidad en el mercado menos competitivo.

De manera similar a Avenali, D’Annunzio y Reverbi (2013), este estudio encuentra que la firma multiproducto aumenta sus precios individuales de manera estratégica, pero su paquete es sin embargo atractivo debido al descuento en sí mismo. La reacción de las firmas monoproducto es reducir sus precios. Los consumidores que intentan comprar otras combinaciones de producto diferentes al paquete 
[A1B1] percibirán que los precios individuales de la firma multiproducto son más altos que los de las firmas monoproducto. Consecuentemente, en el mercado más competitivo, la firma multiproducto encuentra rentable aumentar la inversión en mejoras de la calidad para atraer algunos de esos consumidores. Mientras tanto, la firma monoproducto reduce sus niveles calidad para reducir costos asociados y poder ofrecer precios menores. Éste es un hallazgo muy importante que también es descrito por Avenali, D'Annunzio y Reverberi (2013). Aunque en el presente estudio no se considera el poder de mercado de la firma multiproducto, el empaquetamiento mixto disminuye los incentivos de las firmas a invertir en el contexto de una competencia más intensa. Sin embargo, el resultado en el mercado menos competitivo es ambiguo. Dado que los dos mercados son idénticos en estructura de costos y utilidad adicional de mejoras de calidad y los dos mercados no son tan diferentes en intensidad competitiva ( $\theta$ es lo suficientemente alto), el resultado de las mejoras de calidad es similar a la contraparte del mercado más competitivo.

\section{Proposición 7c}

Después de la implementación de la estrategia de empaquetamiento mixto en vez de la estrategia de no empaquetamiento, un cambio en la participación de mercado es ambiguo.

En el mercado más competitivo, es más probable que la firma multiproducto pueda aumentar su participación de mercado, mientras que la participación de mercado de las firmas monoproducto se reduce.

El resultado en el mercado menos competitivo también es ambiguo. Sin embargo, si los dos mercados son idénticos en su estructura de costos y en su utilidad adicional de mejoras de calidady los costos de degustación no son tan diferentes en los dos mercados ( $\theta$ es lo suficientemente alto), el resultado es similar a los obtenidos en el mercado más competitivo.

Una comparación de participaciones de mercado es ambigua. Si los mercados son idénticos en sus estructuras de costos y utilidad adicional de mejoras de calidad y los dos mercados no son tan diferentes en términos de intensidad competitiva, la estrategia de empaquetamiento mixto no solo afecta los beneficios de las firmas monoproducto, sino también reduce sus participaciones de mercado.

En resumen, la firma multiproducto toma ventaja de un rango amplio de líneas de producto por medio de empaquetamiento mixto para poder obtener más beneficios. Esta puede ofrecer un descuento sobre el paquete como una herramienta adicional de discriminación de precios. Entre tanto, las firmas monoproducto ( $\sin$ cooperación) no pueden implementar esta discriminación de precios y lo mejor que pueden hacer es aplicar un esquema de precios similar a todos los consumidores.

\section{Proposición 7d}

Después de la implementación de la estrategia de empaquetamiento mixto en vez de la estrategia sin empaquetamiento, un cambio en el excedente del consumidor es ambiguo.

Sin embargo, si los dos mercados son idénticos en estructura de costos y utilidad adicional de mejoras en calidad y los costos de degustación de los dos mercados son suficientemente diferentes $(\theta$ es lo suficientemente bajo), el empaquetamiento mixto reduce el bienestar del consumidor.

En el caso extremo analizado por Avenali, D'Annunzio y Reverberi (2013) donde la firma multiproducto ata su componente monopólico con su componente competitivo, la estrategia de empaquetamiento mixto afecta el excedente del consumidor. Sin embargo, el presente estudio relaja el supuesto sobre intensidad competitiva y encuentra que el efecto del empaquetamiento mixto sobre el excedente del consumidor es ambiguo. La estrategia de empaquetamiento mixto, similar a la estrategia de empaquetamiento puro, afecta de manera más probable el bienestar del consumidor cuando los dos mercados unidos son significativamente diferentes en intensidad competitiva. 


\section{Corolario 1}

Suponiendo que ambos mercados son idénticos en costos de degustación, estructura de costos y utilidad adicional de mejoras de calidad, el excedente del consumidor se reduce después del empaquetamiento mixto.

De acuerdo a la proposición $7 \mathrm{~d}$, suponiendo que los dos mercados son significativamente diferentes en los costos de degustación ( $\theta$ es lo suficientemente bajo), los mercados son de manera notable distorsionados después de que el mercado menos competitivo es unido con el mercado más competitivo. Aunque el empaquetamiento mixto puede estimular a las firmas a ofrecer niveles más altos de mejoras de calidad de los productos A1 y B2 en este caso, los cambios en mejoras de calidad de los otros productos tienen un considerable efecto negativo sobre el excedente del consumidor. Como un resultado, el excedente termina reduciéndose. Adicionalmente, suponiendo que los dos mercados no son tan diferentes en costos de degustación ( $\theta$ es lo suficientemente alto), las mejoras de calidad de la firma multipoducto se incrementan levemente mientras que las de las firmas monoproducto se reducen sustancialmente en comparación con el caso simétrico sin empaquetamiento. El efecto de la estrategia de empaquetamiento mixto sobre el excedente del consumidor es ambiguo en este caso. Sin embargo, una caída sustancial en los niveles de mejoras de calidad de las firmas monoproducto es más significativo en comparación con el incremento leve en los niveles de la firma multiproducto. Así que, es probable que el excedente del consumidor se reduzca después del empaquetamiento mixto. El efecto del empaquetamiento mixto sobre el excedente del consumidor es ambiguo de acuerdo a $\theta$. El resultado es ilustrado en los tres casos de $\theta$ ( $\theta$ es bajo, $\theta$ es moderado, y $\theta$ es alto). Sin embargo, este estudio enfoca su análisis de bienestar del consumidor en el caso de un $\theta$ bajo. Esto atrae la atención de los reguladores y está relacionado de manera cercana con la teoría del apalancamiento en el contexto de empaquetamiento.

Como se estableció en el corolario 1 , si los dos mercados son idénticos en costos de degustación, estructura de costos y utilidad adicional de las mejoras de calidad, i.e. $\theta=1, b_{A}=b_{B}, c_{A}=c_{B}, \alpha_{A}=$ $\alpha_{B}$, el resultado de equilibrio en el mercado A es idéntico al del mercado B. Se encuentra que el deterioro de los niveles de calidad de las firmas monoproducto tiene efectos más significativos sobre el excedente del consumidor que la calidad de la firma multiproducto. En este marco de parámetros idénticos, el empaquetamiento mixto reduce el excedente del consumidor.

\section{Conclusiones e implicaciones de política.}

Este estudio examina los efectos del empaquetamiento puro y la estrategia de empaquetamiento mixto sobre los resultados del mercado y el excedente del consumidor. En este modelo, una firma multiproducto y dos rivales monoproducto compiten en dos mercados duopólicos, que tienen diferentes grados de competencia (diferentes costos de degustación). Las firmas ajustan sus niveles de mejoras de calidad y después fijan sus precios para atraer consumidores. Sin empaquetamiento, las firmas compiten la una con la otra en cada mercado separadamente. Esto lleva a un equilibrio simétrico en cada mercado. Sin embargo, ambas estrategias de empaquetamiento dominan la estrategia sin empaquetamiento porque la firma multiproducto puede aumentar beneficios después de empaquetar, en tanto que las firmas monoproducto pierden beneficios en muchas situaciones.

Con empaquetamiento puro, la firma multiproducto considera la externalidad entre los dos mercados interrelacionados, en tanto que sus rivales monoproducto (sin cooperación) la ignoran. Como resultado, la firma multiproducto puede ofrecer niveles más altos de mejoras de calidad que sus rivales. El precio del paquete de la firma multiproducto es más bajo (más alto) que el precio total del producto combinado de las firmas monoproducto si el proceso de mejora de calidad es ineficiente (suficientemente eficiente) en términos de costo y utilidad adicional. 
Sin embargo, comparado con el escenario sin empaquetamiento, el empaquetamiento puro reduce los incentivos de la firma monoproducto a invertir en calidad en ambos mercados, pero la decisión de las firmas monoproducto sobre mejoras en calidad es ambigua. Si el proceso de mejoras de calidad es ineficiente (eficiente) en términos de costo y utilidad adicional, la firma multiproducto reduce (aumenta) sus niveles de mejoras en calidad. La comparación de precios también es ambigua. El precio del paquete de la firma multiproducto y el precio de la firma monoproducto en el mercado más competitivo son más altos que en los escenarios sin empaquetamiento si los grados de competencia de los dos mercados son tan diferentes que estas firmas se benefician de una reducción en la intensidad competitiva después del empaquetamiento puro. Entre tanto, la firma monoproducto en el mercado menos competitivo reduce su precio porque su rival multiproducto es más agresivo con la estrategia de empaquetamiento puro. La participación de mercado de la firma multiproducto aumenta. La firma monoproducto en el mercado menos competitivo experimenta reducción de sus beneficios. A pesar de una reducción en la participación de mercado, la firma monoproducto en el mercado más competitivo puede experimentar un aumento en beneficios si el beneficio de atar el mercado más competitivo con el menos competitivo prevalece.

El empaquetamiento mixto es una estrategia discriminadora de precios. Comparado con el escenario sin empaquetamiento, la firma multiproducto aumenta sus precios individuales en ambos mercados a pesar de su descuento sobre el paquete. Entre tanto, las firmas monoproducto reducen sus precios. En el mercado más competitivo, la firma multiproducto aumenta sus niveles de mejoras de calidad para poder atraer más consumidores a los que se les cobran precios individuales altos. La firma monoproducto reduce sus niveles de calidad en correspondencia a sus menores precios. En el mercado menos competitivo, un cambio en los niveles de calidad es ambiguo. Las participaciones de mercado de la firma multiproducto se pueden aumentar en ambos mercados cuando los dos mercados no son tan diferentes en intesidad competitiva. En adición al empaquetamiento puro, la firma multiproducto también puede implementar la estrategia de empaquetamiento mixto para lograr dominancia bajo estas circunstancias.

Comparado al escenario sin empaquetamiento, la estrategia de empaquetamiento puro reduce el excedente del consumidor cuando los dos mercados son significativamente diferentes en intensidad competidora. En este caso, los efectos negativos de la competencia menos intensa con un limitado número de elecciones de producto sobrepasan los beneficios de la externalidad de la firma multiproducto. De manera similar, el efecto de la estrategia del empaquetamiento mixto sobre el excedente del consumidor es ambiguo. El bienestar del consumidor disminuye si los grados de competencia de los dos mercados son significativamente diferentes. Una reducción en las mejoras de calidad de las firmas monoproducto puede ser más sustancial que un aumento en los niveles de mejoras de calidad de la firma multiproducto. En este caso, la estrategia de empaquetamiento tiene, de manera más probable, un efecto negativo sobre el excedente del consumidor. Similar al empaquetamiento puro, el empaquetamiento mixto tiende a ser empleado para el propósito de aumentar la diferenciación de producto en el mercado más competitivo, además que promueve la competencia en precio y mejoras en calidad, especialmente cuando un mercado más competitivo es unido con un mercado menos competitivo.

El empaquetamiento parece inaceptable cuando el bienestar de un consumidor es la principal preocupación en muchas situaciones. Sin embargo, el regulador sectorial debería tener en cuenta algunos efectos positivos del empaquetamiento. Por ejemplo, el empaquetamiento puro puede promover las mejoras en calidad de la firma multiproducto cuando dichas mejoras no involucran costos relativamente altos. Por lo tanto, en áreas remotas con baja demanda y costos de inversión altos como en el sector de telecomunicaciones, el empaquetamiento puro no es una aproximación apropiada para estimular las mejoras en calidad. El empaquetamiento puro no debería ser permitido en esta situación para poder prevenir prácticas predatorias por la firma multiproducto y así proteger el bienestar del consumidor. De manera opuesta, en mercados más competitivos, el empaquetamiento mixto puede al menos estimular las mejoras en calidad por la firma multiproducto, que de manera probable está sirviendo a la mayoría de los consumidores. La estrategia de empaquetamiento puede allanar el camino para mayores estándares de calidad del producto en el futuro. En adición, los efectos del 
empaquetamiento puro sobre el bienestar del consumidor son todavía ambiguos, así que es posible que el excedente del consumidor pueda aumentar bajo algunas condiciones. Una prohibición de empaquetamiento mixto en esta situación es una intervención miope. Los rivales monoproducto pueden elevar una queja en contra del empaquetamiento en un intento de parar la reducción en sus beneficios. Sin embargo, no es necesario para el regulador intervenir cuando todos los mercados interrelacionados son altamente competitivos.

Investigación adicional puede extenderse a un grupo de consumidores, que intentan consumir ya sea el producto A o el producto B. Adicionalmente, los temas de diferenciación vertical en calidad y segmentación del mercado son también interesantes en el contexto de empaquetamiento.

\section{Referencias}

Armstrong, M. (2011). Bundling revisited:substitute products and inter-firm discounts. Economics Series Working Papers 574, University of Oxford.

Avenali, A., D'Annunzio, A., \& Reverberi, P. (2013). Bundling, competition and quality investment: a welfare analysis. Review of Industrial Organization, 43(3), 221-241.

Choi, J.P. (1996). Preemptive R\&D, rent dissipation, and the "Leverage Theory". Quarterly Journal of Economics, 111(4), 1153-1181.

Choi, J.P. (2004). Tying and innovation: A dynamic analysis of tying arrangements. Economic Journal, 114(492), 83-101.

Gans, J.S., \& King, S.P. (2006). Paying for loyalty: product bundling in oligopoly. Journal of Industrial Economics, 54(1), 43-64.

Granier, L., \& Podesta, M. (2010). Bundling and mergers in energy markets. Energy Economics, 32(6), $1316-1324$.

Heeb, R. (2003). Innovation and vertical integration in complementary markets. Journal of Economics and Management Strategy, 12(3), 387-417.

Krämer, J. (2009). Bundling vertically differentiated communications services to leverage market power. Journal of policy, regulation and strategy for telecom-munications, 11(3), 64-74.

Matutes, C., \& Regibeau, P. (1992). Compatibility and bundling of complementary goods in a duopoly. Journal of Industrial Economics, 40(1), 37-54.

Nalebuff, B. (2004). Bundling as an entry barrier. Quarterly Journal of Economics, 119(1), 159-187.

Reisinger, M. (2006). Product bundling and the correlation of valuations in duopoly. Discussion papers in economics, University of Munich.

Rennhoff, A.D., \& Serfes, K. (2009). The role of upstream-downstream competition on bundling decisions: should regulators force firms to unbundle? Journal of Economics \& Management Strategy, 18(2), 547-588.

Thanassoulis, J. (2007). Competitive mixed bundling and consumer surplus. Journal of Economics \& Management Strategy, 16(2), 437-467. 
Thanassoulis, J. (2011). Is multimedia convergence to be welcomed? Journal of Industrial Economics, $59(2), 225-253$.

Tirole, J. (1988). The theory of industrial organization. MIT press.

Venkatesh, R., \& Kamakura, W. (2003). Optimal bundling and pricing under a monopoly: Contrasting complements and substitutes from independently valued products. Journal of Business, 76(2), 211231.

Wauthy, X. (1996). Quality choice in models of vertical differentiation. Journal of Industrial Economics, 44(3), 345-353. 